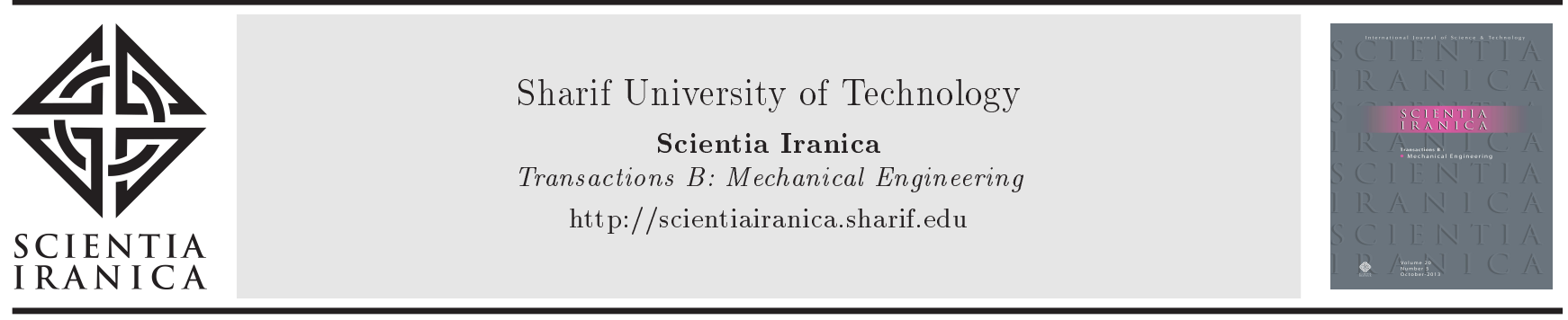

\title{
Effect of hygro-thermal loading on the two-dimensional response of a functionally graded piezomagnetic cylinder under asymmetric loads
}

\author{
M. Gharibavi* and J. Yi \\ Department of Mechanical Engineering, East China University of Science and Technology, Shanghai, China.
}

Received 16 May 2018; received in revised form 26 January 2019; accepted 13 July 2019

KEYWORDS
Complex Fourier
series;
Functionally graded
material;
Piezomagnetic
material;
Asymmetric loading;
Winkler elastic
foundation.

\section{KEYWORDS}

series

Functionally graded

material;

material;

Winkler elastic

foundation.

\begin{abstract}
In this article, a semi-analytical solution is presented in order to analyze a Functionally Graded Piezomagnetic (FGP) cylinder resting on an elastic foundation exposed to hygro-thermal loading. All mechanical, hygro-thermal, and magnetic properties were considered to be varying according to the power-law function through the thickness. The steady-state heat conduction and moisture diffusion equations were employed to attain the moisture concentration and temperature distributions in the FGP cylinder. The constitutive equations as well as magnetic and mechanical equilibrium equations were combined in order to derive three second-order differential equations in terms of magnetic potential and mechanical displacements. Variables were separated and the complex Fourier series method was utilized to solve the governing equations. Numerical results revealed the effects of hygro-thermal loading, elastic foundation, and non-homogeneity constants on hygro-thermo-magneto-elastic response of the FGP cylinder. It was observed that hygrothermal loading had remarkable effects on the behavior of the cylinder, leading to increase in the absolute values of the radial magnetic induction and radial, circumferential, and shear stresses.
\end{abstract}

(C) 2020 Sharif University of Technology. All rights reserved.

\section{Introduction}

Functionally Graded (FG) materials can be distinguished from each other by the compositional gradients of their components. They have high thermal resistance and their thermal and mechanical properties continuously vary with respect to the location. They can be designed for specific functions and applications in many industries, including aerospace, nuclear power, military, medicine, electronics, and biomate-

*. Corresponding author.

E-mail address: m.ghribavi.ecust@gmail.com (M. Gharibavi)

doi: $10.24200 /$ sci.2019.51043.1975 rials. In addition, FG materials with piezoelectric and piezomagnetic properties can be used in ultrasonic transducers as well as many electronic and engineering applications $[1-7]$.

Thick-wall cylindrical vessels are crucial equipment used in oil, chemical, petroleum, petrochemical, and nuclear fields. They are mostly employed in, e.g., power generation by fossil and nuclear fuels, storing gasoline in service stations in the petrochemical industry, and the chemical industry [8]. The thickwall cylinder subjected to multi-physical loadings and surrounded by an elastic foundation is a prime example of soil-structure interaction problems. The static and dynamic analyses of such structures leads to a deeper understanding of the response of the elastic foundation 
under different external loads. The dynamic effects of soil-structure interaction appear in the earthquake response of large-scale structures such as nuclear power plants structures. Furthermore, the study of fracture mechanics, load transfer, and stress concentrations of the mentioned structures is another field of investigation in solid mechanics [9-12].

Many researchers have inquired the mechanical response of smart structures such as FG piezoelectric \piezomagnetic cylinders and spheres under different conditions of loading [13-18]. Hosseini et al. [16] offered a strain gradient elasticity formulation for capturing the size effect in micro-scale structures to analyze the thermo-elastic response of an FG microrotating cylinder. Jabbari et al. [19] presented a general theoretical analysis of the three-dimensional mechanical and thermal stresses in a hollow FG cylinder. They assumed that temperature distribution was a function of radial and circumferential directions and solved the differential equations by employing generalized Bessel function and Fourier series method. Thermopiezo-magneto-elastic response of rotating Functionally Graded Piezomagnetic (FGP) disks exposed to thermal and mechanical loadings was studied by Ghorbanpour Arani et al. [20]. In this study, they explained the effects of non-homogeneity constant on the distributions of stresses, displacement, and magnetic potential. An analytical solution for displacement and the strain and stress field in a rotating thick-wall cylinder made of FG material subjected to the uniform external magnetic and thermal fields was presented by Hosseini and Dini [4]. The magneto-thermo-elastic response of an FG annular sandwich disk was also investigated by Zenkour [21]. Using exp-exp strain energy for modeling the hyperelastic materials, Almasi et al. [22] carried out an analytical and numerical thermo-mechanical study of an FG hyperelastic thick-wall pressure vessel. An elastic-plastic analysis of FG spherical pressure vessels under internal pressure based on strain gradient plasticity was performed in [23]. A feedback gain control algorithm was employed by Barati and Jabbari [24] in the two-dimensional piezothermoelastic analysis of an FG hollow sphere with integrated piezoelectric layers as a sensor and actuator subject to non-axisymmetric loads. They analytically solved the governing equations utilizing the Legendre polynomials and the system of Euler differential equations.

The study of the effects of hygro-thermal loading on the behavior of smart materials and structures has attracted the attention of many researchers in recent years [25-27]. A three-dimensional discrete-layer model was developed by Smittakorn and Heyliger [28] for analyzing rectangular plates, in which the transient and hygro-thermo-piezoelectric responses of plates were evaluated under the coupled effects of mechanical, electrical, thermal, and moisture fields. In another work, Keles and Tutuncu [29] carried out free and forced vibration analysis of FG hollow cylinders and spheres using analytical solutions in which the material properties were assumed to vary based on the power law function. The distributions of the temperature, moisture, displacement, and stress in an FGP circular rotating disk under a coupled hygro-thermal field were studied by Dai et al. [26]. Akbarzadeh and Pasini [30] used a multi-physics model to study the effects of moisture, temperature, magnetic, electric, and mechanical loadings on the response of multi-layer and FG cylinders. Vinyas and Kattimani [31] presented a 3D Finite Element (FE) method in order to analyze a magneto-electro-elastic plate subjected to hygrothermal loads. In their work, an $\mathrm{FE}$ formulation was inferred by employing the principle of total potential energy and linear coupled constitutive equations.

To the best of the authors' knowledge, the study of the effects of hygro-thermal loading and elastic foundation on the stress and strain fields in the FGP cylinders under asymmetric loading is missing in the literature. Hence, using the complex Fourier series method, with the assumption of power-law distribution for FG materials, a cylinder made of piezomagnetic materials embedded in a Winkler elastic foundation under asymmetric hygro-thermo-magneto-mechanical loadings is analyzed in this study.

\section{Preliminaries}

In this section, stress-strain relations and equilibrium equations are expressed and the formulation of the problem is discussed. As shown in Figure 1, a cylinder is considered with the inner and outer radii of $a$ and $b$, which is radially magnetized and exposed to asymmetric hygro-thermal and internal pressure loads. The piezomagnetic cylinder is embedded in a Winklertype elastic foundation with stiffness $k_{w}$. According to the power-law distribution, the material properties for

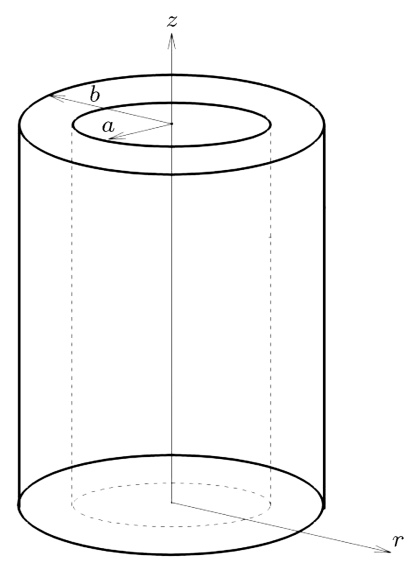

Figure 1. Schematics of the Functionally Graded Piezomagnetic (FGP) cylinder subject to physical loadings. 
the piezomagnetic cylinder are defined as [32]:

$$
\begin{aligned}
& C_{i j}=C_{i j}^{0} r^{\beta_{1}}, \quad d_{i j}=d_{i j}^{0} r^{\beta_{1}}, \quad g_{i j}=\eta_{i j}^{0} r^{\beta_{1}}, \\
& k_{i}=k_{i}^{0} r^{\beta_{3}}, \quad \omega_{i}=\omega_{i}^{0} r^{\beta_{3}}, \quad \lambda_{i}=\lambda_{i}^{0} r^{\beta_{1}+\beta_{2}}, \\
& \mu_{i}=\mu_{i}^{0} r^{\beta_{1}+\beta_{2}},
\end{aligned}
$$

in which $k_{i}$ and $\omega_{i}$ are the thermal conductivity and moisture diffusivity coefficients, respectively. $\beta_{1}, \beta_{2}$, and $\beta_{3}$ are non-homogeneity constants. The strain equations for the cylinder are written as [33]:

$$
\begin{aligned}
& \varepsilon_{r r}=\frac{\partial u}{\partial r}, \quad \varepsilon_{\theta \theta}=\frac{u}{r}+\frac{1}{r} \frac{\partial v}{\partial \theta}, \\
& \varepsilon_{r \theta}=\frac{1}{2}\left[\frac{1}{r} \frac{\partial u}{\partial \theta}+\frac{\partial v}{\partial r}-\frac{v}{r}\right],
\end{aligned}
$$

where $u$ and $v$ are displacements in the radial and circumferential directions, respectively. Magnetic field is considered $B_{i}=-\nabla \psi$ and the constitutive equations are expressed as $[20,32,34]$ :

$$
\begin{aligned}
\sigma_{r r}= & C_{11} \varepsilon_{r r}+C_{12} \varepsilon_{\theta \theta}+d_{11} \psi_{, r}-\vartheta_{1} T(r, \theta) \\
& -\varrho_{1} \bar{M}(r, \theta), \\
\sigma_{\theta \theta}= & C_{12} \varepsilon_{r r}+C_{22} \varepsilon_{\theta \theta}+d_{21} \psi_{, r}-\vartheta_{2} T(r, \theta) \\
& -\varrho_{2} \bar{M}(r, \theta), \\
\tau_{r \theta}= & C_{31} \varepsilon_{r \theta}+C_{12} \varepsilon_{\theta \theta}+d_{31} \frac{1}{r} \psi_{, \theta}, \\
B_{r}= & d_{11} \varepsilon_{r r}+d_{12} \varepsilon_{\theta \theta}-g_{11} \psi_{, r}+q_{1} T(r, \theta)+\gamma_{1} \bar{M}(r, \theta), \\
B_{\theta}= & d_{31} \varepsilon_{r \theta}-g_{22} \frac{1}{r} \psi_{, \theta}+q_{2} T(r, \theta)+\gamma_{2} \bar{M}(r, \theta), \quad(3)
\end{aligned}
$$

in which $\sigma_{i j}, B_{i}, \psi, T$, and $\bar{M}$ are the stress, magnetic induction, magnetic potential, temperature, and moisture concentration, respectively. $C_{i j}, d_{i j}, \vartheta_{i}, \varrho_{i}$, $g_{i j}, q_{i}$, and $\gamma_{i}$ are the elastic, piezomagnetic, thermal stress, hygroscopic stress, magnetic permeability, pyromagnetic, and hygromagnetic coefficients, respectively. The thermal and hygroscopic stresses are associated with the elastic, thermal expansion $\alpha_{i}$, and moisture expansion $\xi_{i}$ coefficients as follows [35,36]:

$$
\begin{array}{ll}
\vartheta_{1}=C_{11} \alpha_{r}+C_{12} \alpha_{\theta}, & \vartheta_{2}=C_{12} \alpha_{r}+C_{22} \alpha_{\theta}, \\
\varrho_{1}=C_{11} \xi_{r}+C_{12} \xi_{\theta}, & \varrho_{2}=C_{12} \xi_{r}+C_{22} \xi_{\theta} .
\end{array}
$$

The mechanical equilibrium equation and Maxwell's electromagnetic relation in the cylindrical coordinate system may be expressed as $[34,37]$ :

$$
\begin{aligned}
& \frac{\partial \sigma_{r r}}{\partial r}+\frac{1}{r} \frac{\partial \tau_{r \theta}}{\partial \theta}+\frac{\sigma_{r r}-\sigma_{\theta \theta}}{r}=0, \\
& \frac{\partial \tau_{r \theta}}{\partial r}+\frac{1}{r} \frac{\partial \sigma_{\theta \theta}}{\partial \theta}+\frac{2}{r} \tau_{r \theta}=0 \\
& \frac{\partial B_{r}}{\partial r}+\frac{1}{r} \frac{\partial B_{\theta}}{\partial \theta}+\frac{B_{r}}{r}=0 .
\end{aligned}
$$

\section{Governing equations}

In this section, the governing equations for the cylinder are given. Submitting Eqs. (2) and (3) into Eq. (5) leads to the coupled differential equations [33,34]:

$$
\begin{aligned}
& C_{11}^{0} r^{2} \frac{\partial^{2} u}{\partial r^{2}}+\left(\beta_{1}+1\right) C_{11}^{0} r \frac{\partial u}{\partial r}+\left(\beta_{1} C_{12}^{0}-C_{22}^{0}\right) u \\
& +C_{31}^{0} \frac{\partial^{2} u}{\partial \theta^{2}}+\left(\beta_{1} C_{12}^{0}-C_{31}^{0}-C_{22}^{0}\right) \frac{\partial v}{\partial \theta} \\
& +\left(C_{12}^{0}+C_{31}^{0}\right) r \frac{\partial^{2} v}{\partial r \partial \theta}+d_{11}^{0} r^{2} \frac{\partial^{2} \psi}{\partial r^{2}} \\
& +d_{31}^{0} \frac{\partial^{2} \psi}{\partial \theta^{2}}+\left[d_{11}^{0}\left(\beta_{1}+1\right)-d_{21}^{0}\right] r \frac{\partial \psi}{\partial r} \\
& +\left[\vartheta_{2}^{0}-\vartheta_{1}^{0}\left(\beta_{1}+\beta_{2}+1\right)\right] r^{\beta_{2}+1} T \\
& -\vartheta_{1}^{0} r^{\beta_{2}+2} \frac{\partial T}{\partial r}+\left[\varrho_{2}^{0}-\varrho_{1}^{0}\left(\beta_{1}+\beta_{2}+1\right)\right] r^{\beta_{2}+1} \bar{M} \\
& -\varrho_{1}^{0} r^{\beta_{2}+2} \frac{\partial \bar{M}}{\partial r}=0, \\
& {\left[C_{31}^{0}\left(\beta_{1}+1\right)+C_{22}^{0}\right] \frac{\partial u}{\partial \theta}+\left(\beta_{1}+1\right) C_{31}^{0} r \frac{\partial v}{\partial r}} \\
& -C_{31}^{0}\left(\beta_{1}+1\right) v+\left(C_{31}^{0}+C_{12}^{0}\right) r \frac{\partial^{2} u}{\partial r \partial \theta} \\
& +C_{31}^{0} r^{2} \frac{\partial^{2} v}{\partial r^{2}}+C_{22}^{0} \frac{\partial^{2} v}{\partial \theta^{2}}+\left(d_{21}^{0}+d_{31}^{0}\right) r \frac{\partial^{2} \psi}{\partial r \partial \theta} \\
& +\left(\beta_{1}+1\right) d_{31}^{0} \frac{\partial \psi}{\partial \theta}-\vartheta_{2}^{0} r^{\beta_{2}+1} \frac{\partial T}{\partial \theta} \\
& -\varrho_{2}^{0} r^{\beta_{2}+1} \frac{\partial \bar{C}}{\partial \theta}=0 \\
& {\left[d_{11}^{0}\left(\beta_{1}+1\right)+d_{21}^{0}\right] r \frac{\partial u}{\partial r}+d_{11}^{0} r^{2} \frac{\partial^{2} u}{\partial r^{2}}+\beta_{1} d_{21}^{0} u} \\
& +\left(\beta_{1} d_{21}^{0}-d_{31}^{0}\right) \frac{\partial v}{\partial \theta}+\left(d_{21}^{0}+d_{31}^{0}\right) r \frac{\partial^{2} v}{\partial r \partial \theta} \\
& +d_{31}^{0} \frac{\partial^{2} u}{\partial \theta^{2}}-g_{11}^{0} r^{2} \frac{\partial^{2} \psi}{\partial r^{2}}-g_{22}^{0} \frac{\partial^{2} \psi}{\partial \theta^{2}} \\
& -\left(\beta_{1}+1\right) g_{11}^{0} r \frac{\partial \psi}{\partial r}+\left(\beta_{1}+\beta_{2}+1\right) q_{1}^{0} r^{\beta_{2}+1} T
\end{aligned}
$$




$$
\begin{aligned}
& +q_{1}^{0} r^{\beta_{2}+2} \frac{\partial T}{\partial r}+q_{2}^{0} r^{\beta_{2}+1} \frac{\partial T}{\partial \theta} \\
& +\left(\beta_{1}+\beta_{2}+1\right) \gamma_{1}^{0} r^{\beta_{2}+1} \bar{M}+\gamma_{1}^{0} r^{\beta_{2}+2} \frac{\partial \bar{M}}{\partial r} \\
& +\gamma_{2}^{0} r^{\beta_{2}+1} \frac{\partial \bar{M}}{\partial \theta}=0 .
\end{aligned}
$$

\section{Hygrothermoelasticity}

The two-dimensional heat conduction and moisture diffusion relations in the cylindrical coordinates can be written as $[36,38,39]$ :

$$
\begin{aligned}
& \frac{1}{r} \frac{\partial}{\partial r}\left(r K_{r} \frac{\partial T}{\partial r}\right)+\frac{1}{r^{2}} \frac{\partial}{\partial \theta}\left(K_{\theta} \frac{\partial T}{\partial \theta}\right)=0, \\
& \frac{1}{r} \frac{\partial}{\partial r}\left(r \omega_{r} \frac{\partial \bar{M}}{\partial r}\right)+\frac{1}{r^{2}} \frac{\partial}{\partial \theta}\left(\omega_{\theta} \frac{\partial \bar{M}}{\partial \theta}\right)=0 .
\end{aligned}
$$

Simplifying Eqs. (9) and (10) yields:

$$
\begin{aligned}
& r^{2} \frac{\partial^{2} T}{\partial r^{2}}+r\left(\beta_{3}+1\right) \frac{\partial T}{\partial r}+\frac{\partial^{2} T}{\partial \theta^{2}}=0, \\
& r^{2} \frac{\partial^{2} \bar{M}}{\partial r^{2}}+r\left(\beta_{3}+1\right) \frac{\partial \bar{M}}{\partial r}+\frac{\partial^{2} \bar{M}}{\partial \theta^{2}}=0 .
\end{aligned}
$$

The complex Fourier series is defined as the solution to Eqs. (11) and (12):

$$
\begin{aligned}
& T(r, \theta)=\sum_{n=-\infty}^{+\infty} T_{n}(r) e^{i n \theta}, \\
& T_{n}(r)=\frac{1}{2 \pi} \int_{-\pi}^{+\pi} T(r, \theta) e^{-i n \theta} d_{\theta}, \\
& \bar{M}(r, \theta)=\sum_{n=-\infty}^{+\infty} \bar{M}_{n}(r) e^{i n \theta}, \\
& \bar{M}_{n}(r)=\frac{1}{2 \pi} \int_{-\pi}^{+\pi} \bar{M}(r, \theta) e^{-i n \theta} d_{\theta},
\end{aligned}
$$

where $T_{n}(r)$ and $\bar{M}_{n}(r)$ are the coefficient of complex Fourier series. By substituting Eqs. (13) and (14) into Eqs. (11) and (12), the following equations are given (please consider revising the distorted text):

$$
\begin{aligned}
& r^{2} \frac{\partial^{2} T_{n}}{\partial r^{2}}+r\left(\beta_{3}+1\right) \frac{\partial T_{n}}{\partial r}-n^{2} T_{n}=0 \\
& r^{2} \frac{\partial^{2} \bar{M}_{n}}{\partial r^{2}}+r\left(\beta_{3}+1\right) \frac{\partial \bar{M}_{n}}{\partial r}-n^{2} \bar{M}_{n}=0 .
\end{aligned}
$$

Using Eqs. (15) and (16), the temperature and moisture distributions in the piezomagnetic cylinder are written as:

$$
\begin{aligned}
& T(r, \theta)=\sum_{n=-\infty}^{+\infty}\left(A_{n 1} r^{m_{n 1}}+A_{n 2} r^{m_{n 2}}\right) e^{i n \theta}, \\
& \bar{M}(r, \theta)=\sum_{n=-\infty}^{+\infty}\left(G_{n 1} r^{S_{n 1}}+G_{n 2} r^{S_{n 2}}\right) e^{i n \theta},
\end{aligned}
$$

in which $A_{n 1}, A_{n 2}, G_{n 1}$, and $G_{n 2}$ are hygro-thermal constants. Also:

$$
\begin{aligned}
& m_{n 1}=\frac{1}{2}\left(-\beta_{3}+\sqrt{\beta_{3}^{2}+4 n^{2}}\right) \\
& m_{n 2}=\frac{1}{2}\left(-\beta_{3}-\sqrt{\beta_{3}^{2}+4 n^{2}}\right) \\
& S_{n 1}=\frac{1}{2}\left(-\beta_{3}+\sqrt{\beta_{3}^{2}+4 n^{2}}\right) \\
& S_{n 2}=\frac{1}{2}\left(-\beta_{3}-\sqrt{\beta_{3}^{2}+4 n^{2}}\right)
\end{aligned}
$$

\section{Solution procedure}

In the complex Fourier series method, the displacements $u(r, \theta)$ and $v(r, \theta)$ and the magnetic potential $\psi(r, \theta)$ are expressed as [40]:

$$
\begin{aligned}
& u(r, \theta)=\sum_{n=-\infty}^{+\infty} u_{n}(r) e^{i n \theta}, \\
& v(r, \theta)=\sum_{n=-\infty}^{+\infty} v_{n}(r) e^{i n \theta}, \\
& \psi(r, \theta)=\sum_{n=-\infty}^{+\infty} \psi_{n}(r) e^{i n \theta},
\end{aligned}
$$

where:

$$
\begin{aligned}
& u_{n}(r)=\frac{1}{2 \pi} \int_{-\pi}^{+\pi} u(r, \theta) e^{-i n \theta} d_{\theta}, \\
& v_{n}(r)=\frac{1}{2 \pi} \int_{-\pi}^{+\pi} v(r, \theta) e^{-i n \theta} d_{\theta}, \\
& \psi_{n}(r)=\frac{1}{2 \pi} \int_{-\pi}^{+\pi} \psi(r, \theta) e^{-i n \theta} d_{\theta} .
\end{aligned}
$$

By submitting Eq. (20) into Eqs. (6)-(8), three differential equations can be derived: 


$$
\begin{array}{ccc}
C_{11}^{0} r^{2} u_{n}^{\prime \prime}+\left(\beta_{1}+1\right) C_{11}^{0} r u_{n}^{\prime} & \text { in which } B, C, \text { and } D \text { are unknown constants deter- } \\
& +\left(\beta_{1} C_{12}^{0}-C_{22}^{0}-n^{2} C_{31}^{0}\right) u_{n} & \begin{array}{c}
\text { mined by boundary conditions. Submitting Eq. (25) } \\
\text { into Eqs. }(22)-(24) \text { yields: }
\end{array} \\
+\left(\beta_{1} C_{12}^{0}-C_{31}^{0}-C_{22}^{0}\right)(i n) v_{n} & {\left[C_{11}^{0} \zeta\left(\beta_{1}+\zeta\right)+\left(\beta_{1} C_{12}^{0}-C_{22}^{0}-n^{2} C_{31}^{0}\right)\right] B} \\
+\left(C_{12}^{0}+C_{31}^{0}\right)(i n) r v_{n}^{\prime}+d_{11}^{0} r^{2} \psi_{n}^{\prime \prime} & +\left[\left(\beta_{1} C_{12}^{0}-C_{31}^{0}-C_{22}^{0}\right)(i n)+\left(C_{12}^{0}+C_{31}^{0}\right)(i n) \zeta\right] C \\
+\left[d_{11}^{0}\left(\beta_{1}+1\right)-d_{21}^{0}\right] r \psi_{n}^{\prime}-n^{2} d_{31}^{0} \psi_{n} & +\left[d_{11}^{0} \zeta\left(\beta_{1}+\zeta\right)-d_{21}^{0} \zeta-n^{2} d_{31}^{0}\right] D=0 \\
=\left[\vartheta_{1}^{0}\left(\beta_{1}+\beta_{2}+m_{n 1}+1\right)-\vartheta_{2}^{0}\right] A_{n 1} r^{m_{n 1}+\beta_{2}+1} & {\left[\left(C_{31}^{0}+C_{12}^{0}\right)(i n) \zeta+\left(C_{31}^{0}\left(\beta_{1}+1\right)+C_{22}^{0}\right)(i n)\right] B} \\
+\left[\vartheta_{1}^{0}\left(\beta_{1}+\beta_{2}+m_{n 2}+1\right)-\vartheta_{2}^{0}\right] A_{n 2} r^{m_{n 2}+\beta_{2}+1} & +\left[C_{31}^{0} \zeta\left(\beta_{1}+\zeta\right)-\left(n^{2} C_{22}^{0}+\left(\beta_{1}+1\right) C_{31}^{0}\right)\right] C \\
+\left[\varrho_{1}^{0}\left(\beta_{1}+\beta_{2}+S_{n 1}+1\right)-\varrho_{2}^{0}\right] G_{n 1} r^{S_{n 1}+\beta_{2}+1} & +\left[\left(d_{31}^{0}+d_{21}^{0}\right)(i n) \zeta+\left(\beta_{1}+1\right)(i n) d_{31}^{0}\right] D=0, \\
+\left[\varrho_{1}^{0}\left(\beta_{1}+\beta_{2}+S_{n 2}+1\right)-\varrho_{2}^{0}\right] G_{n 2} r^{S_{n 2}+\beta_{2}+1},(22) & {\left[d_{11}^{0} \zeta\left(\beta_{1}+\zeta\right)+d_{21}^{0} \zeta+\left(\beta_{1} d_{21}^{0}-n^{2} d_{31}^{0}\right)\right] B} \\
\left(C_{31}^{0}+C_{12}^{0}\right)(i n) r u_{n}^{\prime}+\left[C_{31}^{0}\left(\beta_{1}+1\right)+C_{22}^{0}\right](i n) u_{n} & +\left[\left(d_{21}^{0}+d_{31}^{0}\right)(i n) \zeta+\left(\beta_{1} d_{21}^{0}-d_{31}^{0}\right)(i n)\right] C
\end{array}
$$$$
+C_{31}^{0} r^{2} v_{n}^{\prime \prime}+\left(\beta_{1}+1\right) C_{31}^{0} r v_{n}^{\prime}
$$$$
-\left[n^{2} C_{22}^{0}+\left(\beta_{1}+1\right) C_{31}^{0}\right] v_{n}+\left(d_{31}^{0}+d_{21}^{0}\right)(i n) r \psi_{n}^{\prime}
$$$$
+\left(\beta_{1}+1\right)(i n) d_{31}^{0} \psi_{n}
$$$$
=\vartheta_{2}^{0}(i n)\left[A_{n 1} r^{m_{n 1}+\beta_{2}+1}+A_{n 2} r^{m_{n 2}+\beta_{2}+1}\right]
$$$$
+\varrho_{2}^{0}(i n)\left[G_{n 1} r^{S_{n 1}+\beta_{2}+1}+G_{n 2} r^{S_{n 2}+\beta_{2}+1}\right],
$$$$
d_{11}^{0} r^{2} u_{n}^{\prime \prime}+\left[\left(\beta_{1}+1\right) d_{11}^{0}+d_{21}^{0}\right] r u_{n}^{\prime}
$$$$
+\left(\beta_{1} d_{21}^{0}-n^{2} d_{31}^{0}\right) u_{n}+\left(d_{21}^{0}+d_{31}^{0}\right)(i n) r v_{n}^{\prime}
$$$$
+\left(\beta_{1} d_{21}^{0}-d_{31}^{0}\right)(i n) v_{n}-g_{11}^{0} r^{2} \psi_{n}^{\prime \prime}
$$$$
-\left(\beta_{1}+1\right) g_{11}^{0} r \psi_{n}^{\prime}+g_{22}^{0} n^{2} \psi_{n}
$$$$
=-\left[q_{1}^{0}\left(\beta_{1}+\beta_{2}+m_{n 1}+1\right)+(i n) q_{2}^{0}\right] A_{n 1} r^{m_{n 1}+\beta_{2}+1}
$$$$
-\left[q_{1}^{0}\left(\beta_{1}+\beta_{2}+m_{n 2}+1\right)+(i n) q_{2}^{0}\right] A_{n 1} r^{m_{n 1}+\beta_{2}+1}
$$$$
-\left[q_{1}^{0}\left(\beta_{1}+\beta_{2}+m_{n 2}+1\right)+(i n) q_{2}^{0}\right] A_{n 2} r^{m_{n 2}+\beta_{2}+1}
$$$$
-\left[\gamma_{1}^{0}\left(\beta_{1}+\beta_{2}+S_{n 1}+1\right)+(i n) \gamma_{2}^{0}\right] G_{n 1} r^{S_{n 1}+\beta_{2}+1}
$$$$
-\left[\gamma_{1}^{0}\left(\beta_{1}+\beta_{2}+S_{n 2}+1\right)+(i n) \gamma_{2}^{0}\right] G_{n 2} r^{S_{n 2}+\beta_{2}+1}
$$

The symbols "'" and " " " indicate the first and second derivatives with respect to the variable $r$, respectively. Eqs. (22)-(24) are ordinary differential equations to which the general solution can be formulated as:

$$
u_{n}^{g}(r)=B r^{\zeta}, \quad v_{n}^{g}(r)=C r^{\zeta}, \quad \psi_{n}^{g}(r)=D r^{\zeta},
$$

The determinant of the system in Eq. (27) should be equal to zero in order to attain a nontrivial solution to Eq. (26). Therefore, six roots $\zeta_{n j}(j=1,2, \ldots, 6)$ of Eq. (27) are achieved:

$$
\begin{aligned}
& u_{n}(r)=\sum_{j=1}^{6} B_{n j} r^{\zeta_{n j}}, \\
& v_{n}(r)=\sum_{j=1}^{6} L_{n j} B_{n j} r^{\zeta_{n j}}, \\
& \psi_{n}(r)=\sum_{j=1}^{6} P_{n j} B_{n j} r^{\zeta_{n j}},
\end{aligned}
$$

where $L_{n j}$ is the relation between constants $B_{n j}$ and $C_{n j}$, and $P_{n j}$ is the relation between constants $B_{n j}$ and $D_{n j}$ calculated by Eq. (26) as:

$$
\begin{aligned}
& L_{n j}=\frac{b_{1} \cdot b_{6}-b_{4} \cdot b_{3}}{b_{3} \cdot b_{5}-b_{2} \cdot b_{6}}, \quad P_{n j}=\frac{b_{8} \cdot b_{1}-b_{7} \cdot b_{2}}{b_{9} \cdot b_{2}-b_{3} \cdot b_{8}}, \\
& j=1,2, \cdots, 6, \\
& b_{1}=C_{11}^{0} \zeta_{n j}\left(\beta_{1}+\zeta_{n j}\right)+\left(\beta_{1} C_{12}^{0}-C_{22}^{0}-n^{2} C_{31}^{0}\right), \\
& b_{2}=\left(\beta_{1} C_{12}^{0}-C_{31}^{0}-C_{22}^{0}\right)(i n)+\left(C_{12}^{0}+C_{31}^{0}\right)(i n) \zeta_{n j}, \\
& b_{3}=d_{11}^{0} \zeta_{n j}\left(\beta_{1}+\zeta_{n j}\right)-d_{21}^{0} \zeta_{n j}-n^{2} d_{31}^{0}, \\
& b_{4}=\left(C_{31}^{0}+C_{12}^{0}\right)(i n) \zeta_{n j}+\left(C_{31}^{0}\left(\beta_{1}+1\right)+C_{22}^{0}\right)(i n), \\
& b_{5}=C_{31}^{0} \zeta_{n j}\left(\beta_{1}+\zeta_{n j}\right)-\left(n^{2} C_{22}^{0}+\left(\beta_{1}+1\right) C_{31}^{0}\right), \\
& b_{6}=\left(d_{31}^{0}+d_{21}^{0}\right)(i n) \zeta_{n j}+\left(\beta_{1}+1\right)(i n) d_{31}^{0}, \\
& b_{7}=d_{11}^{0} \zeta_{n j}\left(\beta_{1}+\zeta_{n j}\right)+d_{21}^{0} \zeta_{n j}+\left(\beta_{1} d_{21}^{0}-n^{2} d_{31}^{0}\right),
\end{aligned}
$$




$$
\begin{aligned}
& b_{8}=\left(d_{21}^{0}+d_{31}^{0}\right)(i n) \zeta_{n j}+\left(\beta_{1} d_{21}^{0}-d_{31}^{0}\right)(i n), \\
& b_{9}=-g_{11}^{0} \zeta_{n j}\left(\beta_{1}+\zeta_{n j}\right)+g_{22}^{0} n^{2}
\end{aligned}
$$

The particular solutions $u_{n}^{p}(r), v_{n}^{p}(r)$, and $\psi_{n}^{p}(r)$ are as follows:

$$
\begin{aligned}
u_{n}^{p}(r)= & Q_{n}^{1} r^{m_{n 1}+\beta_{2}+1}+Q_{n}^{2} r^{m_{n 2}+\beta_{2}+1} \\
& +Q_{n}^{3} r^{S_{n 1}+\beta_{2}+1}+Q_{n}^{4} r^{S_{n 2}+\beta_{2}+1} \\
v_{n}^{p}(r)= & Q_{n}^{5} r^{m_{n 1}+\beta_{2}+1}+Q_{n}^{6} r^{m_{n 2}+\beta_{2}+1} \\
& +Q_{n}^{7} r^{S_{n 1}+\beta_{2}+1}+Q_{n}^{8} r^{S_{n 2}+\beta_{2}+1} \\
\psi_{n}^{p}(r)= & Q_{n}^{9} r^{m_{n 1}+\beta_{2}+1}+Q_{n}^{10} r^{m_{n 2}+\beta_{2}+1} \\
& +Q_{n}^{11} r^{S_{n 1}+\beta_{2}+1}+Q_{n}^{12} r^{S_{n 2}+\beta_{2}+1}
\end{aligned}
$$

$Q_{n}^{j}(j=1,2, \cdots, 12)$ are constants. By substituting Eq. (30) into Eqs. (22)-(24) and equating the coefficients of the identical powers, $r^{S_{n 1}+\beta_{2}+1}, r^{S_{n 2}+\beta_{2}+1}$, $r^{S_{n 1}+\beta_{2}+1}$, and $r^{S_{n 2}+\beta_{2}+1}$, we have:

$$
\begin{aligned}
& \bar{X}_{1} Q_{n}^{1}+\bar{X}_{2} Q_{n}^{5}+\bar{X}_{3} Q_{n}^{9}=\bar{Y}_{1}, \\
& \bar{X}_{4} Q_{n}^{1}+\bar{X}_{5} Q_{n}^{5}+\bar{X}_{6} Q_{n}^{9}=\bar{Y}_{2}, \\
& \bar{X}_{7} Q_{n}^{1}+\bar{X}_{8} F_{n}^{5}+\bar{X}_{9} Q_{n}^{9}=\bar{Y}_{3}, \\
& \bar{X}_{10} Q_{n}^{2}+\bar{X}_{11} Q_{n}^{6}+\bar{X}_{12} Q_{n}^{10}=\bar{Y}_{4}, \\
& \bar{X}_{13} Q_{n}^{2}+\bar{X}_{14} Q_{n}^{6}+\bar{X}_{15} Q_{n}^{10}=\bar{Y}_{5}, \\
& \bar{X}_{16} Q_{n}^{2}+\bar{X}_{17} Q_{n}^{6}+\bar{X}_{18} Q_{n}^{10}=\bar{Y}_{6}, \\
& \bar{X}_{19} Q_{n}^{3}+\bar{X}_{20} Q_{n}^{7}+\bar{X}_{21} Q_{n}^{11}=\bar{Y}_{7}, \\
& \bar{X}_{22} Q_{n}^{3}+\bar{X}_{23} Q_{n}^{7}+\bar{X}_{24} Q_{n}^{11}=\bar{Y}_{8}, \\
& \bar{X}_{25} Q_{n}^{3}+\bar{X}_{26} Q_{n}^{7}+\bar{X}_{27} Q_{n}^{11}=\bar{Y}_{9}, \\
& \bar{X}_{28} Q_{n}^{4}+\bar{X}_{29} Q_{n}^{8}+\bar{X}_{30} Q_{n}^{12}=\bar{Y}_{10} \\
& \bar{X}_{31} Q_{n}^{4}+\bar{X}_{32} Q_{n}^{8}+\bar{X}_{33} Q_{n}^{12}=\bar{Y}_{11}, \\
& \bar{X}_{34} Q_{n}^{4}+\bar{X}_{35} Q_{n}^{8}+\bar{X}_{36} Q_{n}^{12}=\bar{Y}_{12}
\end{aligned}
$$

The constants $\bar{X}_{i}(i=1,2, \ldots, 36)$ and $\bar{Y}_{j}(j=$ $1,2, \ldots, 12)$ are presented in the appendix. The constants $Q_{n}^{j}(j=1,2, \ldots, 12)$ are evaluated by the solution to Eqs. (31)-(34) as a system of algebraic equations. Utilizing Eqs. (27) and (30), we have:

$$
\begin{aligned}
& u_{n}(r)=\left(\sum_{j=1}^{6} B_{n j} r^{\zeta_{n j}}\right)+Q_{n}^{1} r^{m_{n 1}+\beta_{2}+1} \\
& +Q_{n}^{2} r^{m_{n 2}+\beta_{2}+1}+Q_{n}^{3} r^{S_{n 1}+\beta_{2}+1}+Q_{n}^{4} r^{S_{n 2}+\beta_{2}+1} \\
& v_{n}(r)=\left(\sum_{j=1}^{6} L_{n j} B_{n j} r^{\zeta_{n j}}\right)+Q_{n}^{5} r^{m_{n 1}+\beta_{2}+1} \\
& +Q_{n}^{6} r^{m_{n 2}+\beta_{2}+1}+Q_{n}^{7} r^{S_{n 1}+\beta_{2}+1}+Q_{n}^{8} r^{S_{n 2}+\beta_{2}+1} \\
& \psi_{n}(r)=\left(\sum_{j=1}^{6} P_{n j} B_{n j} r^{\zeta_{n j}}\right)+Q_{n}^{9} r^{m_{n 1}+\beta_{2}+1} \\
& +Q_{n}^{10} r^{m_{n 2}+\beta_{2}+1}+Q_{n}^{11} r^{S_{n 1}+\beta_{2}+1}+Q_{n}^{12} r^{S_{n 2}+\beta_{2}+1}
\end{aligned}
$$

For $n=0$, the coefficients $L_{n j}$ and $P_{n j}$ are undefined. Substituting $n=0$ into Eqs. (22)-(24) and following the solution procedure, the complete solutions for $u_{0}(r), v_{0}(r)$, and $\phi_{0}(r)$ are derived as:

$$
\begin{aligned}
& u_{0}(r)=\left(\sum_{j=1}^{4} B_{0 j} r^{\zeta_{0 j}}\right)+Q_{0}^{1} r^{m_{01}+\beta_{2}+1} \\
& +Q_{0}^{2} r^{m_{02}+\beta_{2}+1}+Q_{0}^{3} r^{S_{01}+\beta_{2}+1}+Q_{0}^{4} r^{S_{02}+\beta_{2}+1} \\
& v_{0}(r)=\sum_{j=5}^{6} B_{0 j} r^{\zeta_{0 j}}, \\
& \psi_{0}(r)=\left(\sum_{j=1}^{4} P_{0 j} B_{0 j} r^{\zeta_{0 j}}\right)+Q_{0}^{9} r^{m_{01}+\beta_{2}+1} \\
& +Q_{0}^{10} r^{m_{02}+\beta_{2}+1}+Q_{0}^{11} r^{S_{01}+\beta_{2}+1}+Q_{0}^{12} r^{S_{02}+\beta_{2}+1}
\end{aligned}
$$

where:

$$
P_{0 j}=-\frac{\left[C_{11}^{0} \zeta_{0 j}\left(\zeta_{0 j}+\beta_{1}\right)+\beta_{1} C_{12}^{0}-C_{22}^{0}\right]}{\left[d_{11}^{0} \zeta_{0 j}\left(\zeta_{0 j}+\beta_{1}\right)-d_{21}^{0} \zeta_{0 j}\right]} .
$$

$\zeta_{05}$ and $\zeta_{06}$ can be written as:

$$
\begin{gathered}
\zeta_{05}=\frac{-\beta_{1}+\sqrt{\beta_{1}^{2}+4\left(\beta_{1}+1\right)}}{2}, \\
\zeta_{06}=\frac{-\beta_{1}-\sqrt{\beta_{1}^{2}+4\left(\beta_{1}+1\right)}}{2} .
\end{gathered}
$$

Therefore, the radial and circumferential displacements and magnetic potential are approximated by the sum of Eqs. (35) and (36): 


$$
\begin{aligned}
& u(r, \theta)=\sum_{n=-\infty, n \neq 0}^{\infty}\left\{\left(\sum_{j=1}^{6} B_{n j} r^{\zeta_{n j}}\right)\right. \\
& +Q_{n}^{1} r^{m_{n 1}+\beta_{2}+1}+Q_{n}^{2} r^{m_{n 2}+\beta_{2}+1}+Q_{n}^{3} r^{S_{n 1}+\beta_{2}+1} \\
& \left.+Q_{n}^{4} r^{S_{n 2}+\beta_{2}+1}\right\} e^{i n \theta}+\left(\sum_{j=1}^{4} B_{0 j} r^{\zeta_{0 j}}\right) \\
& +Q_{0}^{1} r^{m_{01}+\beta_{2}+1}+Q_{0}^{2} r^{m_{02}+\beta_{2}+1}+Q_{0}^{3} r^{S_{01}+\beta_{2}+1} \\
& +Q_{0}^{4} r^{S_{02}+\beta_{2}+1}, \\
& +Q_{0}^{12} r^{S_{02}+\beta_{2}+1} . \\
& +Q_{n}^{9} r^{m_{01}+\beta_{2}+1}+Q_{0}^{10} r^{m_{02}+\beta_{2}+1}+Q_{0}^{11} r^{S_{01}+\beta_{2}+1} \\
& +\sum_{n=-\infty, n \neq 0}^{\infty}\left\{\left(\sum_{j=1}^{6} L_{n j} B_{n j} r^{\zeta_{n j}}\right)\right. \\
& +Q_{n}^{5} r^{m_{n 1}+\beta_{2}+1}+Q_{n}^{6} r^{m_{n 2}+\beta_{2}+1}+Q_{n}^{7} r^{S_{n 1}+\beta_{2}+1} \\
& \left.+Q_{n}^{8} r^{S_{n 2}+\beta_{2}+1}\right\} e^{i n \theta}+\left(\sum_{j=5}^{6} B_{0 j} r^{\zeta_{0 j}}\right)
\end{aligned}
$$

The displacement potential field of an FG piezoelectric solid has been addressed in [41]. Submitting Eqs. (39)(41), (17), and (18) into Eq. (3), the components of stress and magnetic induction are attained as:

$$
\begin{aligned}
& \sigma_{r r}(r, \theta)=\sum_{n=-\infty, n \neq 0}^{\infty}\left\{\left(\sum_{j=1}^{6} Z_{n j}^{1} B_{n j} r^{\zeta_{n j}+\beta_{1}-1}\right)\right. \\
& +\bar{W}_{n}^{1} r^{m_{n 1}+\beta_{1}+\beta_{2}}+\bar{W}_{n}^{2} r^{m_{n 2}+\beta_{1}+\beta_{2}} \\
& \left.\quad+\bar{W}_{n}^{3} r^{S_{n 1}+\beta_{1}+\beta_{2}}+\bar{W}_{n}^{4} r^{S_{n 2}+\beta_{1}+\beta_{2}}\right\} e^{i n \theta} \\
& \quad+\left(\sum_{j=1}^{4} Z_{0 j}^{1} B_{0 j} r^{\zeta_{0 j}+\beta_{1}-1}\right)+\bar{W}_{0}^{1} r^{m_{01}+\beta_{1}+\beta_{2}}
\end{aligned}
$$

$$
\begin{aligned}
& +\bar{W}_{0}^{2} r^{m_{02}+\beta_{1}+\beta_{2}}+\bar{W}_{0}^{3} r^{S_{01}+\beta_{1}+\beta_{2}} \\
& +\bar{W}_{0}^{4} r^{S_{02}+\beta_{1}+\beta_{2}}
\end{aligned}
$$

$$
\begin{aligned}
& \sigma_{\theta \theta}(r, \theta)=\sum_{n=-\infty, n \neq 0}^{\infty}\left\{\left(\sum_{j=1}^{6} Z_{n j}^{2} B_{n j} r^{\zeta_{n j}+\beta_{1}-1}\right)\right. \\
& +\bar{W}_{n}^{5} r^{m_{n 1}+\beta_{1}+\beta_{2}}+\bar{W}_{n}^{6} r^{m_{n 2}+\beta_{1}+\beta_{2}} \\
& \left.+\bar{W}_{n}^{7} r^{S_{n 1}+\beta_{1}+\beta_{2}}+\bar{W}_{n}^{8} r^{S_{n 2}+\beta_{1}+\beta_{2}}\right\} e^{i n \theta} \\
& +\left(\sum_{j=1}^{4} Z_{0 j}^{2} B_{0 j} r^{\zeta_{0 j}+\beta_{1}-1}\right)+\bar{W}_{0}^{5} r^{m_{01}+\beta_{1}+\beta_{2}} \\
& +\bar{W}_{0}^{6} r^{m_{02}+\beta_{1}+\beta_{2}}+\bar{W}_{0}^{7} r^{S_{01}+\beta_{1}+\beta_{2}} \\
& +\bar{W}_{0}^{8} r^{S_{02}+\beta_{1}+\beta_{2}},
\end{aligned}
$$

$$
\begin{aligned}
& \tau_{r \theta}(r, \theta)=\sum_{n=-\infty, n \neq 0}^{\infty}\left\{\left(\sum_{j=1}^{6} Z_{n j}^{3} B_{n j} r^{\zeta_{n j}+\beta_{1}-1}\right)\right. \\
& +\bar{W}_{n}^{9} r^{m_{n 1}+\beta_{1}+\beta_{2}}+\bar{W}_{n}^{10} r^{m_{n 2}+\beta_{1}+\beta_{2}} \\
& \left.+\bar{W}_{n}^{11} r^{S_{n 1}+\beta_{1}+\beta_{2}}+\bar{W}_{n}^{12} r^{S_{n 2}+\beta_{1}+\beta_{2}}\right\} e^{i n \theta} \\
& \quad+\left(\sum_{j=5}^{6} Z_{0 j}^{3} B_{0 j} r^{\zeta_{0 j}+\beta_{1}-1}\right),
\end{aligned}
$$

$$
\begin{aligned}
& B_{r}(r, \theta)=\sum_{n=-\infty, n \neq 0}^{\infty}\left\{\left(\sum_{j=1}^{6} Z_{n j}^{4} B_{n j} r^{\zeta_{n j}+\beta_{1}-1}\right)\right. \\
& +\bar{W}_{n}^{13} r^{m_{n 1}+\beta_{1}+\beta_{2}}+\bar{W}_{n}^{14} r^{m_{n 2}+\beta_{1}+\beta_{2}} \\
& \left.+\bar{W}_{n}^{15} r^{S_{n 1}+\beta_{1}+\beta_{2}}+\bar{W}_{n}^{16} r^{S_{n 2}+\beta_{1}+\beta_{2}}\right\} e^{i n \theta} \\
& +\left(\sum_{j=1}^{4} Z_{0 j}^{4} B_{0 j} r^{\zeta_{0 j}+\beta_{1}-1}\right)+\bar{W}_{0}^{9} r^{m_{01}+\beta_{1}+\beta_{2}} \\
& +\bar{W}_{0}^{10} r^{m_{02}+\beta_{1}+\beta_{2}}+\bar{W}_{0}^{11} r^{S_{01}+\beta_{1}+\beta_{2}} \\
& +\bar{W}_{0}^{12} r^{S_{02}+\beta_{1}+\beta_{2}},
\end{aligned}
$$




$$
\begin{aligned}
B_{\theta}(r, \theta)= & \sum_{n=-\infty, n \neq 0}^{\infty}\left\{\left(\sum_{j=1}^{6} Z_{n j}^{5} B_{n j} r^{\zeta_{n j}+\beta_{1}-1}\right)\right. \\
& +\bar{W}_{n}^{17} r^{m_{n 1}+\beta_{1}+\beta_{2}}+\bar{W}_{n}^{18} r^{m_{n 2}+\beta_{1}+\beta_{2}} \\
& \left.+\bar{W}_{n}^{19} r^{S_{n 1}+\beta_{1}+\beta_{2}}+\bar{W}_{n}^{20} r^{S_{n 2}+\beta_{1}+\beta_{2}}\right\} e^{i n \theta} \\
& +\left(\sum_{j=5}^{6} Z_{0 j}^{5} B_{0 j} r^{\zeta_{0 j}+\beta_{1}-1}\right)
\end{aligned}
$$

where constants $Z_{0 j}^{i}, Z_{n j}^{i}(i=1,2, \ldots, 5), \bar{W}_{n}^{i}(i=$ $1,2, \ldots, 20)$, and $\bar{W}_{0}^{i}(i=1,2 \cdots 12)$ are defined in the Appendix. It is noteworthy that Eqs. (42)-(46) consist of six unknown constants $B_{n j},(j=1,2, \ldots, 6)$ and six boundary conditions are needed to approximate $B_{n j}$.

\section{Numerical results and discussion}

\subsection{Verification of the presented solution}

To verify the solution, the results are depicted in Figure 2 for the axisymmetric FGP cylinder, regardless of moisture concentration effects (i.e., $\bar{M}=0$ ). Given the assumption of axisymmetric loadings (i.e., $\theta=0)$, three coupled differential equations, namely, Eqs. (22)-(24), derived in this study are reduced to two coupled differential equations, as stated in [20]. For this purpose, the geometrical parameters used are $a=0.2 \mathrm{~m}$ and $b=1 \mathrm{~m}$ and the non-homogeneity constant is assumed to be $\beta=1$. Also, the boundary conditions are set to $\sigma_{r r}(r=a)=\sigma_{r r}(r=b)=$ 0 . The thermal, magnetic, and mechanical properties are chosen following Ghorbanpour Arani et al. [20].

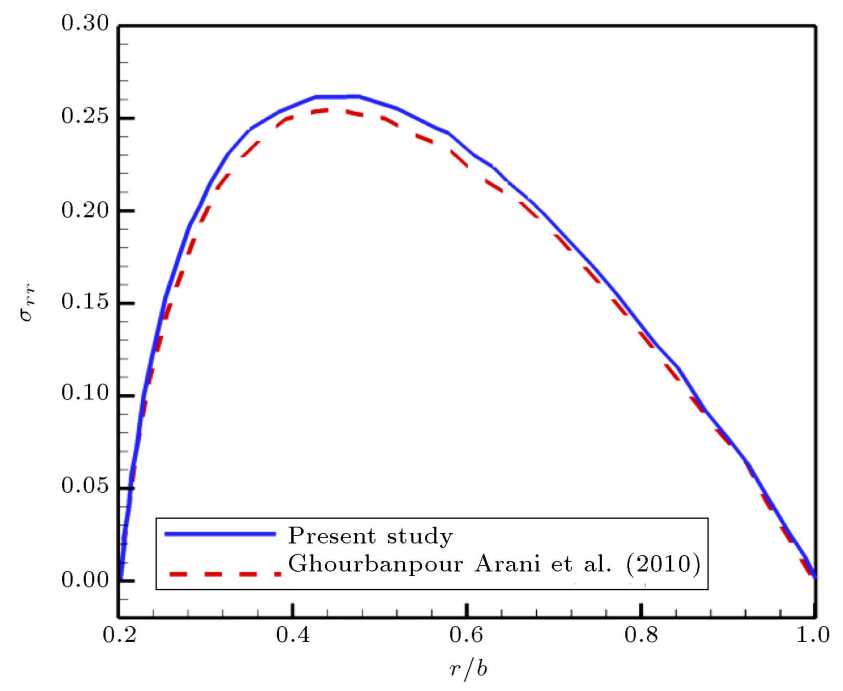

Figure 2. Distribution of the radial stress. Comparison between the present study and Ref. [20].
Figure 2 shows the distribution of the radial stress for the purpose of verification. It can be observed that the results of the present study have a good agreement with those given in [20].

\subsection{Current results}

In this section, numerical results of the analysis of the FGP cylinder resting on an elastic foundation are discussed. The distributions of stress, displacement, magnetic potential, temperature, and moisture are illustrated. The inner and outer radii of the piezomagnetic cylinder are assumed $a=1 \mathrm{~m}$ and $b=1.2 \mathrm{~m}$, respectively. The material properties of $\mathrm{BaTiO}_{3} / \mathrm{CoFe}_{2} \mathrm{O}_{4}$ are listed in Table $1[36,37,42]$. The boundary conditions on the inner and outer surfaces of the piezomagnetic cylinder are expressed as follows:

$$
\begin{array}{ll}
T(a, \theta)=60 \cos 2 \theta, & T(b, \theta)=100 \cos 2 \theta(\mathrm{K}), \\
\bar{M}(a, \theta)=\cos 2 \theta, & \bar{M}(b, \theta)=3 \cos 2 \theta\left(\mathrm{kg} / \mathrm{m}^{3}\right), \\
\sigma_{r r}(a, \theta)=10 \cos 2 \theta, & \sigma_{r r}(b, \theta)=-k_{w} u(b, \theta)(\mathrm{MPa}), \\
\tau_{r \theta}(a, \theta)=0, & \tau_{r \theta}(b, \theta)=0(\mathrm{MPa}), \\
\psi(a, \theta)=10^{4} \cos 2 \theta, & \psi(b, \theta)=0(\mathrm{~W} / \mathrm{A}) .
\end{array}
$$

Figures 3 and 4 reveal the distributions of moisture concentration and temperature, respectively. As shown, the boundary conditions are satisfied at the inner and outer surfaces of the FGP cylinder. Also, the maximum values of moisture concentration and temperature occur at the angles of $\theta=0, \pm \pi$.

Table 1. Material properties of $\mathrm{BaTiO}_{3} / \mathrm{CoFe}_{2} \mathrm{O}_{4}$ [36,37,42].

\begin{tabular}{lc}
$C_{11}^{0}(\mathrm{GPa})$ & 269.5 \\
$C_{12}^{0}(\mathrm{GPa})$ & 170.5 \\
$C_{22}^{0}(\mathrm{GPa})$ & 286 \\
$C_{31}^{0}(\mathrm{GPa})$ & 170.5 \\
$d_{11}^{0}(\mathrm{~N} / \mathrm{Am})$ & 699.7 \\
$d_{21}^{0}(\mathrm{~N} / \mathrm{Am})$ & 580.3 \\
$d_{31}^{0}(\mathrm{~N} / \mathrm{Am})$ & 550 \\
$g_{11}^{0}\left(10^{-4} \mathrm{Ns}^{2} / \mathrm{C}^{2}\right)$ & 1.57 \\
$g_{22}^{0}\left(10^{-4} \mathrm{Ns}^{2} / \mathrm{C}^{2}\right)$ & -5.9 \\
$\alpha_{r}\left(10^{-6} 1 / \mathrm{K}\right)$ & 10 \\
$\alpha_{\theta}\left(10^{-6} 1 / \mathrm{K}\right)$ & 10 \\
$\xi_{r}\left(10^{-4} \mathrm{~m}^{3} / \mathrm{kg}\right)$ & 0.8 \\
$\xi_{\theta}\left(10^{-4} \mathrm{~m}^{3} / \mathrm{kg}\right)$ & 1.1 \\
$q_{1}^{0}\left(10^{-3} \mathrm{~N} / \mathrm{AmK}\right)$ & 6 \\
$q_{2}^{0}\left(10^{-3} \mathrm{~N} / \mathrm{AmK}\right)$ & 6 \\
$\gamma_{1}^{0}\left(10^{-5} \mathrm{Nm}^{2} / \mathrm{Akg}\right)$ & 0 \\
$\gamma_{2}^{0}\left(10^{-5} \mathrm{Nm}^{2} / \mathrm{Akg}\right)$ & 0 \\
\hline
\end{tabular}




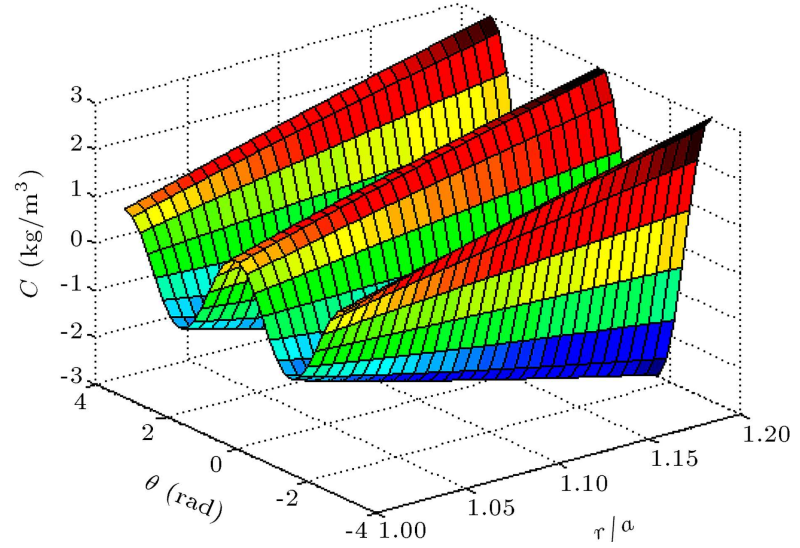

Figure 3. Distribution of moisture concentration in the Functionally Graded Piezomagnetic (FGP) cylinder.

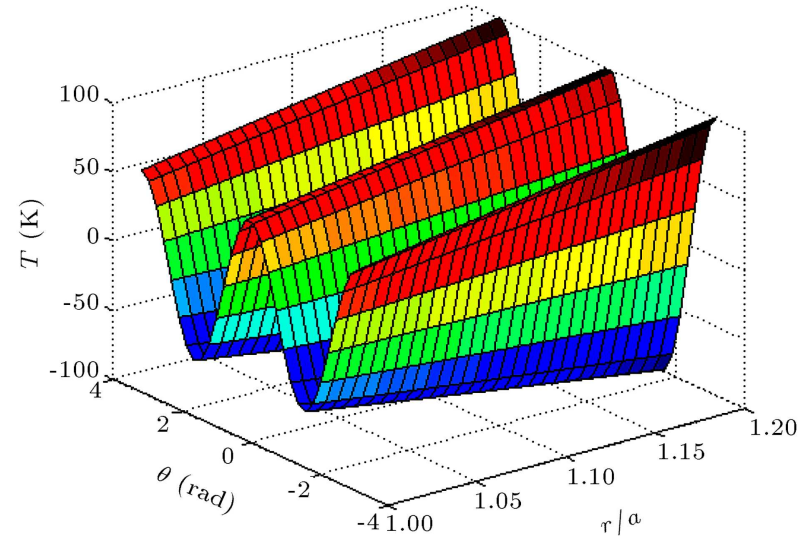

Figure 4. Distribution of temperature in the Functionally Graded Piezomagnetic (FGP) cylinder.

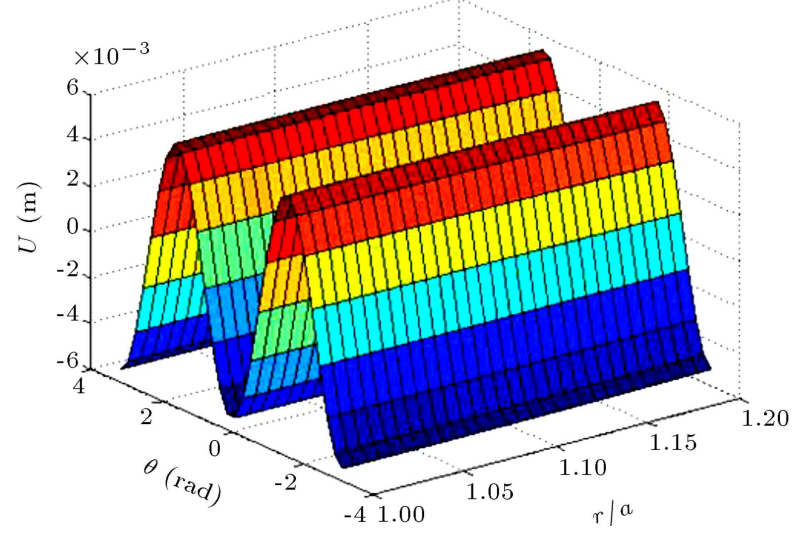

Figure 5. Distribution of radial displacement in the Functionally Graded Piezomagnetic (FGP) cylinder.

Figures 5-9 illustrate the distributions of the radial and circumferential displacement and radial, circumferential, and shear stresses, respectively. It is observed in Figure 5 that the minimum values of the radial displacement occur at the angles of $\theta=0, \pm \pi$, whereas the critical values are achieved at the angles of $\theta= \pm \frac{\pi}{2}$.

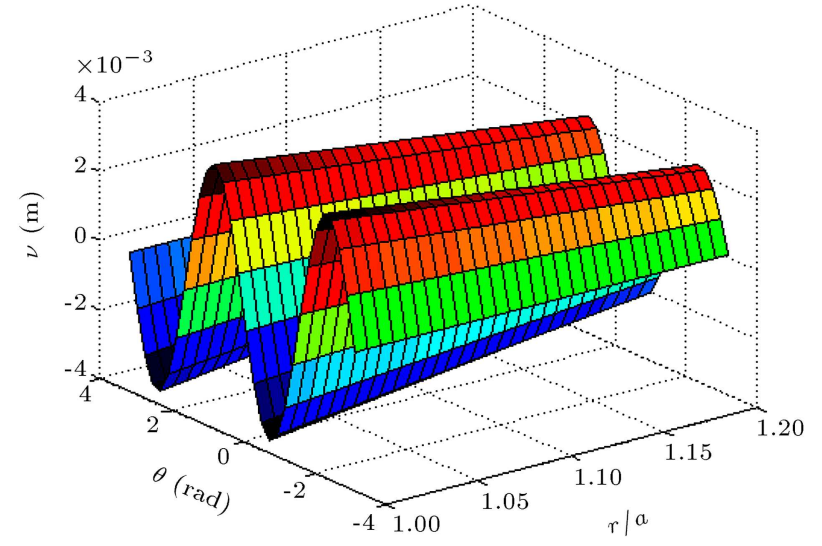

Figure 6. Distribution of circumferential displacement in the Functionally Graded Piezomagnetic (FGP) cylinder.

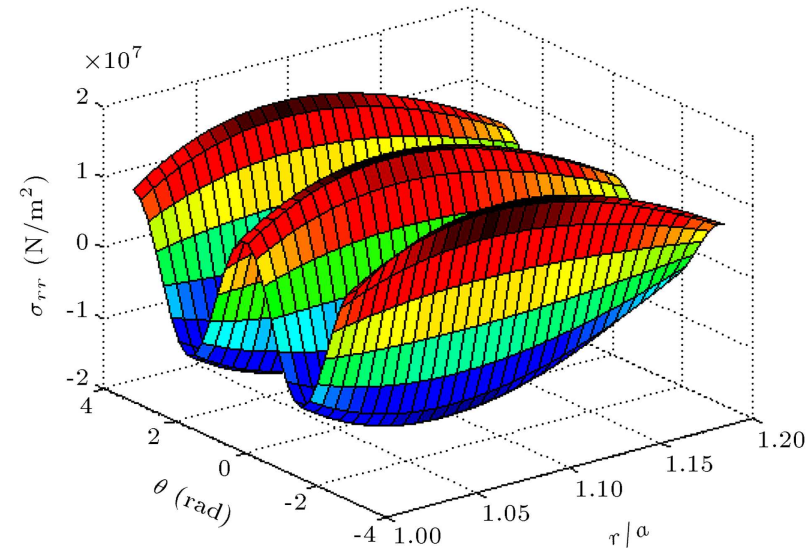

Figure 7. Distribution of radial stress in the Functionally Graded Piezomagnetic (FGP) cylinder.

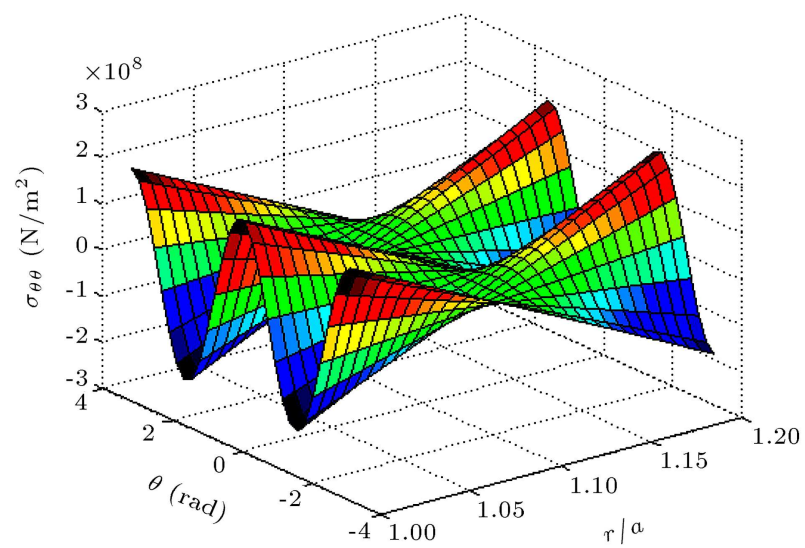

Figure 8. Distribution of circumferential stress in the Functionally Graded Piezomagnetic (FGP) cylinder.

It is evident in Figures 7 and 9 that the boundary conditions of radial and shear stresses are satisfied at the inner and outer surfaces of the cylinder. As depicted, the maximum values of radial stress are achieved at the angles of $\theta=0, \pm \pi$, and the values of shear stress are equal to zero at the angles of $\theta=$ $0, \pm \pi$. It can be seen in Figure 8 that the behavior of 


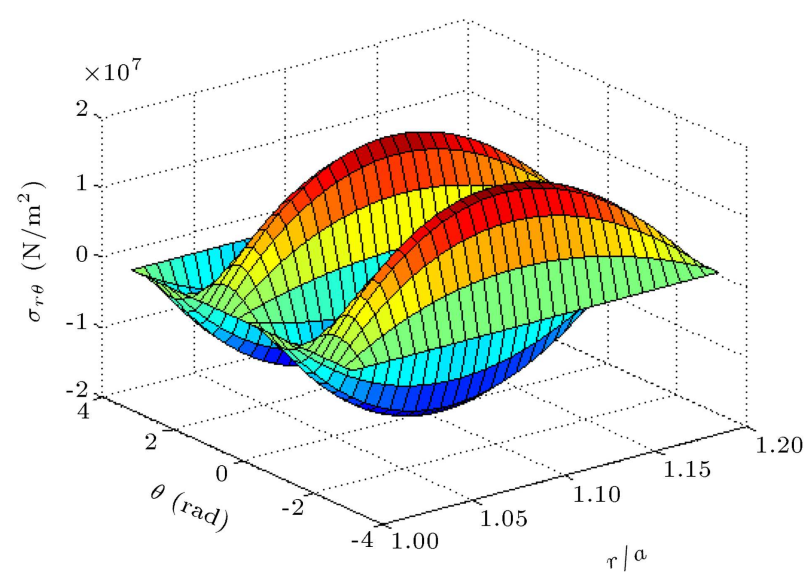

Figure 9. Distribution of shear stress in the Functionally Graded Piezomagnetic (FGP) cylinder.

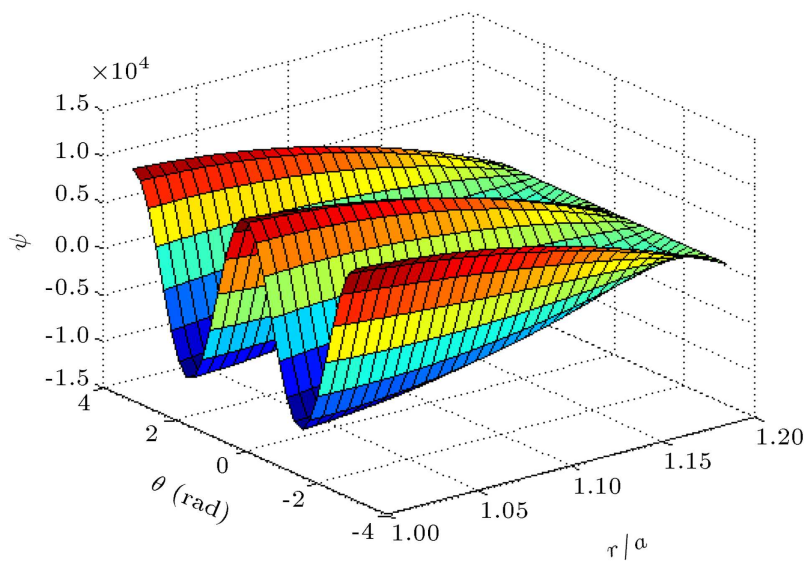

Figure 10. Distribution of magnetic potential in the Functionally Graded Piezomagnetic (FGP) cylinder.

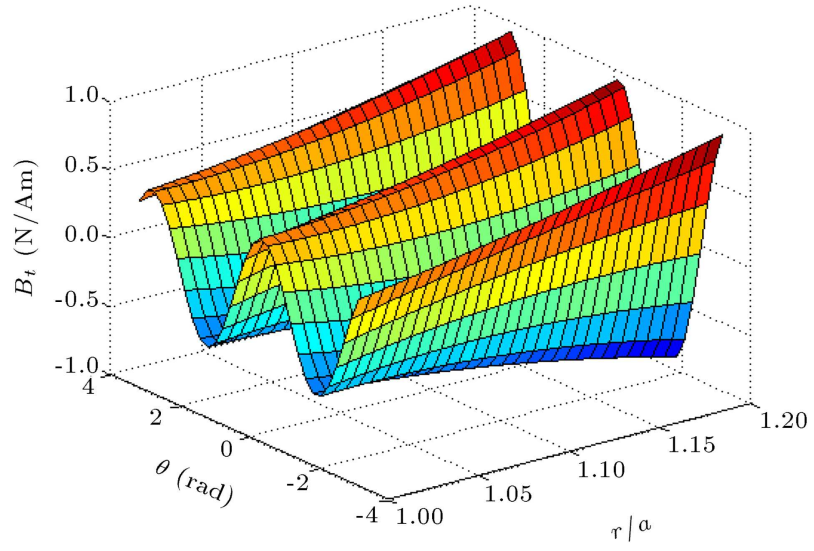

Figure 11. Distribution of radial magnetic induction in the Functionally Graded Piezomagnetic (FGP) cylinder.

the circumferential stress is reversed at the radius of $r / a>1.1$. The distributions of the magnetic potential and radial and circumferential magnetic inductions are depicted in Figures 10-12. As shown, the maximum values occur at the angles of $\theta=0, \pm \pi$, whereas the minimum values arise at $\theta= \pm \frac{\pi}{2}$.

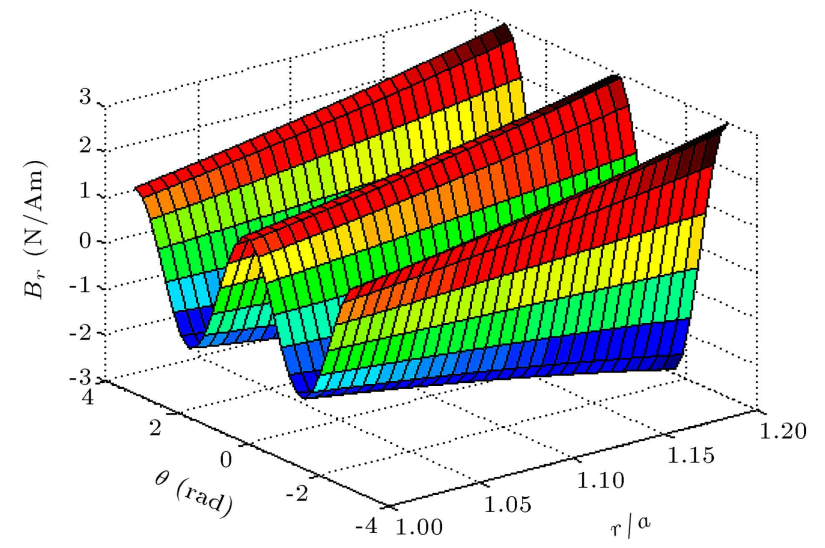

Figure 12. Distribution of circumferential magnetic induction in the Functionally Graded Piezomagnetic (FGP) cylinder.

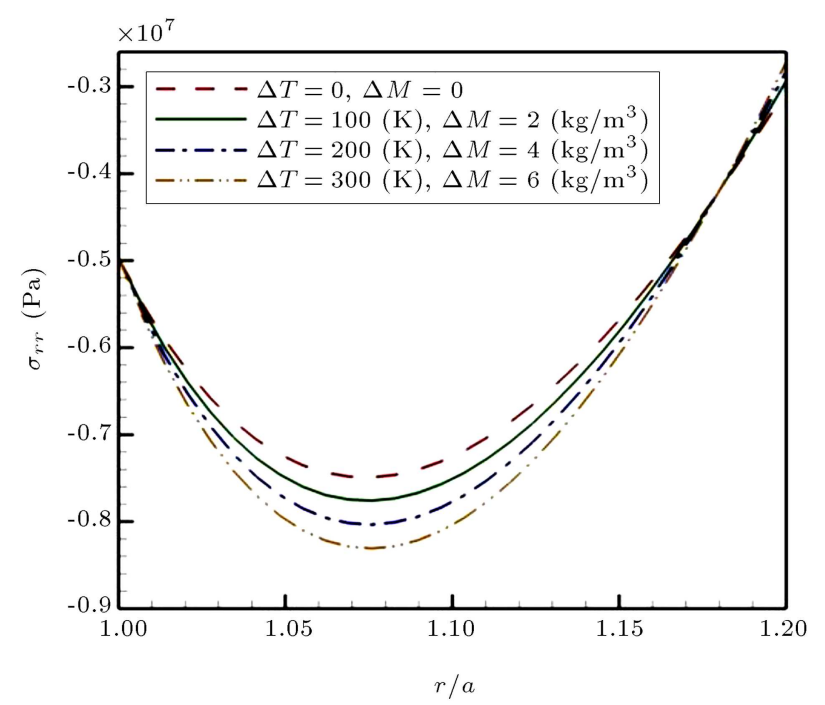

Figure 13. Distribution of radial stress with different hygrothermal loadings $\left(\theta=\pi / 3, \beta=0.5, k_{w}=10^{9} \mathrm{~N} / \mathrm{m}^{3}\right)$.

The effects of hygro-thermal loading on the response of the piezomagnetic cylinder at $\theta=\pi / 3$ are presented in Figures 13-16, wherein the nonhomogeneity constants and foundation stiffness are considered as $\beta_{1}=\beta_{2}=\beta_{3}=\beta=0.5$ and $k_{w}=$ $10^{9}\left(\mathrm{~N} / \mathrm{m}^{3}\right)$ respectively. Figures $13-16$ are depicted on the basis of the temperature difference $\Delta T=T_{o}-T_{i}$ and moisture difference $\Delta \bar{M}=\bar{M}_{o}-\bar{M}_{i}$. Figure 13 indicates that increase in the hygro-thermal loading enhances the absolute values of radial stress. As shown in Figure 14, increase in the hygro-thermal loading raises the absolute values of circumferential stress. It can be seen in Figure 15 that the absolute values of shear stress increase by raising the hygro-thermal loading. As shown in Figure 16, raising the hygrothermal loading leads to an increase in the absolute values of radial magnetic induction. Therefore, it can be concluded that hygro-thermal loading has negative effects on the response of a thick-wall structure. 


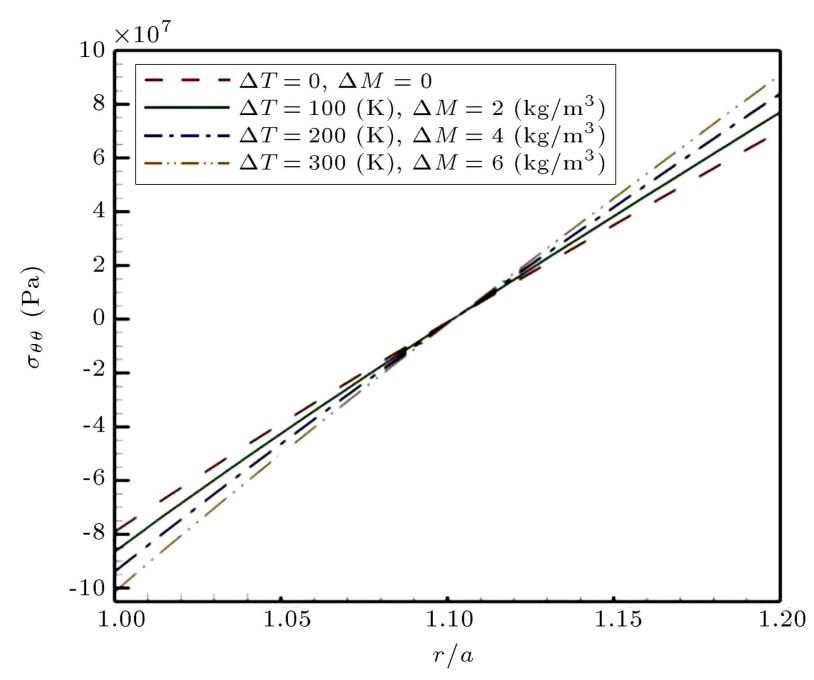

Figure 14. Distribution of circumferential stress with different hygrothermal loadings $(\theta=\pi / 3, \beta=0.5$, $\left.k_{w}=10^{9} \mathrm{~N} / \mathrm{m}^{3}\right)$.

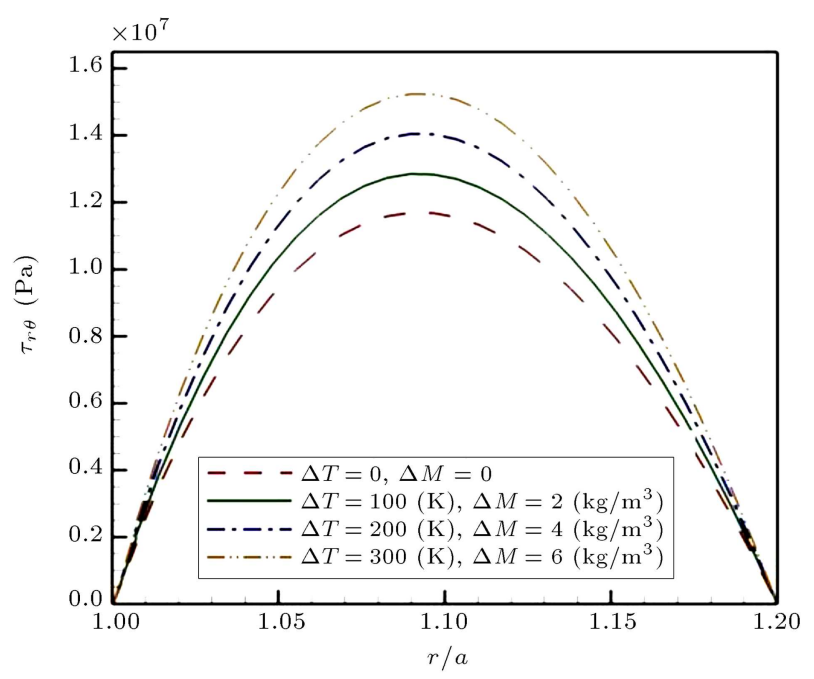

Figure 15. Distribution of shear stress with different hygrothermal loadings $\left(\theta=\pi / 3, \beta=0.5, k_{w}=10^{9} \mathrm{~N} / \mathrm{m}^{3}\right)$.

\section{Conclusion}

In the current study, complex Fourier series method was employed to assess the hygro-thermo-magnetoelastic response of the piezomagnetic cylinders made of Functionally Graded (FG) materials resting on an elastic foundation. The material properties were considered based on the power-law function. The coupled differential equations in terms of mechanical displacements and magnetic potential were solved using the separation of variables and complex Fourier series method. The main advantage of this method is that any type of mechanical and magnetic boundary conditions can be defined in it without any limitations. Numerical results obtained in this study were evaluated to investigate the effects of hygro-thermal loading. It should be pointed out that in the uncoupled

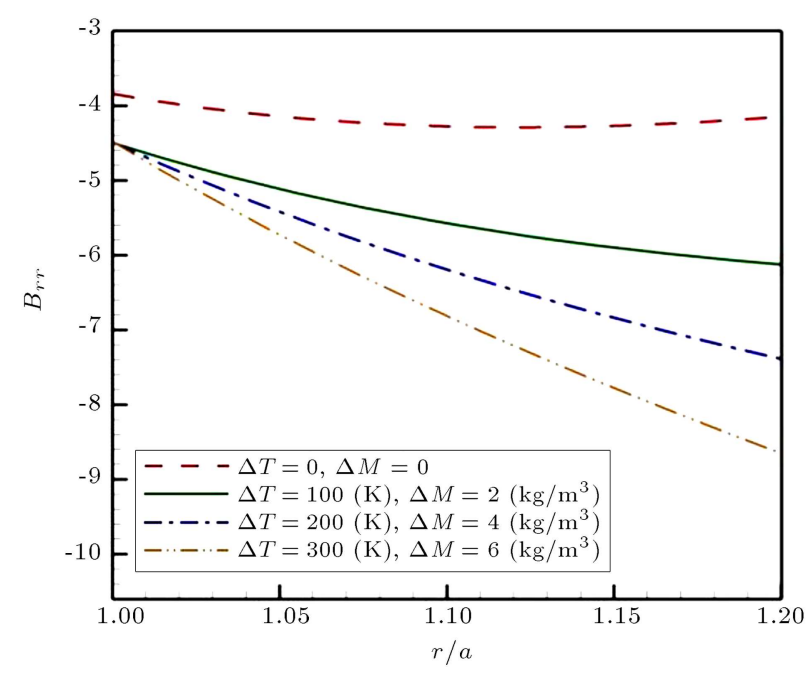

Figure 16. Distribution of magnetic induction with different hygrothermal loadings $(\theta=\pi / 3, \beta=0.5$, $k_{w}=10^{9} \mathrm{~N} / \mathrm{m}^{3}$ ).

hygrothemal problems, the temperature and moisture concentration have not only the same manner, but also similar effects on the behavior of the cylinder. As observed in the results, all the components of stress, displacement, magnetic potential, and magnetic induction followed a harmonic pattern in the cross section of the piezomagnetic cylinder. Furthermore, hygrothermal loading had considerable effects on the stresses and magnetic induction distributions. Moreover, the absolute values of the radial, circumferential, and shear stresses and radial magnetic induction increased by raising the hygrothermal loading.

\section{Nomenclature}

$\begin{array}{ll}\sigma_{i j} & \text { Stress components }(\mathrm{Pa}) \\ \varepsilon_{i j} & \text { Strain components } \\ B_{i} & \text { Magnetic induction (N/Am) } \\ u & \text { Radial displacement }(\mathrm{m}) \\ v & \text { Circumferential displacement }(\mathrm{m}) \\ T & \text { Temperature }(\mathrm{K}) \\ M & \text { Moisture concentration }\left(\mathrm{kg} / \mathrm{m}^{3}\right) \\ \psi & \text { Magnetic potential }(\mathrm{Nm} / \mathrm{C}) \\ C_{i j} & \text { Elastic coefficient }(\mathrm{Pa}) \\ d_{i j} & \text { Piezomagnetic coefficient }(\mathrm{N} / \mathrm{Am}) \\ \vartheta_{i} & \text { Thermal stress }\left(\mathrm{N} / \mathrm{m}^{2} \mathrm{~K}\right) \\ \varrho_{i} & \text { Hygroscopic stress }(\mathrm{Nm} / \mathrm{kg}) \\ g_{i j} & \text { Magnetic permeability }\left(\mathrm{Ns}{ }^{2} / \mathrm{C}^{2}\right) \\ \alpha_{i} & \text { Thermal expansion coefficient }(1 / \mathrm{K}) \\ \xi_{i} & \text { Moisture expansion coefficient }\left(\mathrm{m}^{3} / \mathrm{kg}\right) \\ q_{i} & \text { Pyromagnetic coefficient }(\mathrm{N} / \mathrm{AmK})\end{array}$




$\begin{array}{ll}\gamma_{i} & \text { Hygromagnetic coefficient }\left(\mathrm{Nm}^{2} / \mathrm{Akg}\right) \\ \omega_{i} & \text { Moisture diffusivity }\left(\mathrm{kg} / \mathrm{ms}^{\circ} \mathrm{M}\right) \\ k_{i} & \text { Thermal conductivity }(\mathrm{W} / \mathrm{mK}) \\ \beta_{i} & \text { Non-homogeneity constant } \\ k_{w} & \text { Elastic foundation stiffness }\end{array}$

\section{References}

1. Koizumi, M. "FGM activities in Japan", Composites: Part B, 28, pp. 1-4 (1996).

2. Miyamoto, Y., Kaysser, W., Rabin, B., Kawasaki, A., and Ford, R., Functionally Graded Materials: Design, Processing and Appllications, Kluwer Academic Publishers (1999).

3. Mahamood, R.M.A., Esther, T., Shukla, M., and Pityana, S. "Functionally graded material: An overview", Proceedings of the World Congress on Engineering, 3, pp. 1-5 (2012).

4. Hosseini, M. and Dini, A. "Magneto-thermo-elastic response of a rotating functionally graded cylinder", Structural Engineering and Mechanics, 56(1), pp. 137156 (2015).

5. Mohsenizadeh, M., Gasbarri, F., Munther, M., Beheshti, A., and Davami, K. "Additively-manufactured lightweight metamaterials for energy absorption", $M a$ terials \& Design, 139, pp. 521-530 (2018).

6. Rao, S.S. and Sunar, M. "Piezoelectricity and its use in disturbance sensing and control of flexible structures: A survey", Applied Mechanics Reviews, 47(4), pp. 113-123 (1994).

7. Davami, K., Mohsenizadeh, M., Munther, M., Palma, T., Beheshti, A., and Momeni, K. "Dynamic energy absorption characteristics of additively-manufactured shape-recovering lattice structures", Mat. Res. Express., 6(4), p. 045302 (2018).

8. Chattopadhyay, S., Pressure Vessels: Design and Practice, CRC Press (2004).

9. Mita, A. and Kumagai, S.J.F. "An experimental study on soil-structure interaction effects", FAPIG (Tokyo), pp. 51-59 (1989).

10. Ahmadi, S.F. and Eskandari, M. "Rocking rotation of a rigid disk embedded in a transversely isotropic halfspace", Civil. Eng. Infrastruct. J., 47(1), pp. 125-138 (2014).

11. Ahmadi, S.F. and Eskandari, M. "Vibration analysis of a rigid circular disk embedded in a transversely isotropic solid", J. Eng. Mech., 140(7), p. 04014048 (2013).

12. Pak, R.Y. and Gobert, A.T. "Forced vertical vibration of rigid discs with arbitrary embedment", J. Eng. Mech., 117(11), pp. 2527-2548 (1991).

13. Khoshgoftar, M.J., Mirzaali, M.J., and Rahimi, G.H. "Thermoelastic analysis of non-uniform pressurized functionally graded cylinder with variable thickness using first order shear deformation theory (FSDT) and perturbation method", Chinese Journal of Mechanical Engineering, 28(6), pp. 1149-1156 (2015).

14. Atrian, A., Jafari Fesharaki, J., and Nourbakhsh, S.H. "Thermo-electromechanical behavior of functionally graded piezoelectric hollow cylinder under nonaxisymmetric loads", Applied Mathematics and $\mathrm{Me}$ chanics, 36(7), pp. 939-954 (2015).

15. Grigorenko, A.Y., Müller, W.H., Wille, R., and Loza, I.A. "Nonaxisymmetric electroelastic vibrations of a hollow sphere made of functionally gradient piezoelectric material", Continuum Mechanics and Thermodynamics, 26(6), pp. 771-781 (2014).

16. Hosseini, M., Dini, A., and Eftekhari, M. "Strain gradient effects on the thermoelastic analysis of a functionally graded micro-rotating cylinder using generalized differential quadrature method", Acta Mechanica, 228(5), pp. 1563-1580 (2017).

17. Meshkini, M., Firoozbakhsh, K., Jabbari, M., and Selkghafari, A. "Steady state thermal and mechanical stresses in two-dimensional functionally graded piezoelectric materials (2D-FGPMs) hollow cylinder", $J$. Scientia Iranica, 26(1), pp. 428-444 (2019).

18. Pourhamid, R., Ahmadian, M.T., Mahdavy Moghaddam, H., and Mohammadzadeh, A.R. "Mechanical analysis of a functionally graded cylinder-piston under internal pressure due to a combustion engine using a cylindrical super element and considering thermal loading", J. Scientia Iranica, Transactions B, Mechanical Engineering, 22(2), pp. 493-503 (2015).

19. Jabbari, M., Mohazzab, A.H., Bahtui, A., and Eslami, M.R. "Analytical solution for three-dimensional stresses in a short length FGM hollow cylinder", Zamm, 87(6), pp. 413-429 (2007).

20. Ghorbanpour Arani, A., Khoddami Maraghi, Z., Mozdianfard, M.R., and Shajari, A.R. "Thermo-piezomagneto-mechanical stresses analysis of FGPM hollow rotating thin disk", Int. J. Mech. Mater. Des., 6(4), pp. 341-349 (2011).

21. Zenkour, A.M. "On the magneto-thermo-elastic responses of FG annular sandwich disks", International Journal of Engineering Science, 75, pp. 54-66 (2014).

22. Almasi, A., Baghani, M., Moallemi, A., Baniassadi, M., and Faraji, G. "Investigation on thermal stresses in FGM hyperelastic thick-walled cylinders", Journal of Thermal Stresses, 41(2), pp. 204-221 (2018/02/01 2018).

23. Shokrollahi, H. "Elastic-plastic analysis of functionally graded spherical pressure vessels using strain gradient plasticity", International Journal of Applied Mechanics, 9(8), p. 1750118 (2017).

24. Barati, A.R. and Jabbari, M. "Two-dimensional piezothermoelastic analysis of a smart FGM hollow sphere", Acta Mechanica, 226(7), pp. 2195-2224 (2015).

25. Sayman, O. "Analysis of multi-layered composite cylinders under hygrothermal loading", Composites Part A: Applied Science and Manufacturing, 36(7), pp. 923-933 (2005). 
26. Dai, H.-L., Zheng, Z.-Q., and Dai, T. "Investigation on a rotating FGPM circular disk under a coupled hygrothermal field", Applied Mathematical Modelling, 46, pp. 28-47 (2017).

27. Ebrahimi, F. and Barati, M.R. "Hygrothermal effects on vibration characteristics of viscoelastic FG nanobeams based on nonlocal strain gradient theory", Composite Structures, 159, pp. 433-444 (2017).

28. Smittakorn, W. and Heyliger, P.R. "A discrete-layer model of laminated hygrothermopiezoelectric plates", Mechanics of Advanced Materials and Structures, $\mathbf{7}(1)$, pp. 79-104 (2000).

29. Keles, I. and Tutuncu, N. "Exact analysis of axisymmetric dynamic response of functionally graded cylinders (or disks) and spheres", Journal of Applied Mechanics, 78(6), p. 061014 (2011).

30. Akbarzadeh, A.H. and Pasini, D. "Multiphysics of multilayered and functionally graded cylinders under prescribed hygrothermomagnetoelectromechanical loading", Journal of Applied Mechanics, 81(4), p. 041018 (2013).

31. Vinyas, M. and Kattimani, S.C. "Hygrothermal analysis of magneto-electro-elastic plate using 3D finite element analysis", Composite Structures, 180, pp. 617-637 (2017).

32. Akbarzadeh, A.H. and Chen, Z.T. "Hygrothermal stresses in one-dimensional functionally graded piezoelectric media in constant magnetic field", Composite Structures, 97, pp. 317-331 (2013).

33. Jafari Fesharaki, J., Jafari Fesharaki, V., Yazdipoor, M., and Razavian, B. "Two-dimensional solution for electro-mechanical behavior of functionally graded piezoelectric hollow cylinder", Applied Mathematical Modelling, 36(11), pp. 5521-5533 (2012).

34. Alashti, R.A. and Khorsand, M. "Three-dimensional thermo-elastic analysis of a functionally graded cylindrical shell with piezoelectric layers by differential quadrature method", International Journal of Pressure Vessels and Piping, 88(5-7), pp. 167-180 (2011).

35. Saadatfar, M. and Aghaie-Khafri, M. "Hygrothermomagnetoelectroelastic analysis of a functionally graded magnetoelectroelastic hollow sphere resting on an elastic foundation", Smart Materials and Structures, 23(3), p. 035004 (2014).

36. Saadatfar, M. and Aghaie-Khafri, M. "Hygrothermal analysis of a rotating smart exponentially graded cylindrical shell with imperfect bonding supported by an elastic foundation", Aerospace Science and Technology, 43, pp. 37-50 (2015).

37. Akbarzadeh, A.H. and Chen, Z.T. "Magnetoelectroelastic behavior of rotating cylinders resting on an elastic foundation under hygrothermal loading", Smart Materials and Structures, 21(12), p. 125013 (2012).

38. Sih, G.C., Michopoulos, J., and Chou, S.C., Hygrothermoelasticity, Dordrecht: Martinus Nijhoff Publishers (1986).
39. Zenkour, A.M. "Hygrothermoelastic responses of inhomogeneous piezoelectric and exponentially graded cylinders", International Journal of Pressure Vessels and Piping, 119, pp. 8-18 (2014).

40. Badrieh, F. "Complex Fourier series", In Spectral, Convolution and Numerical Techniques in Circuit Theory, Springer, pp. 125-134 (2018).

41. Samea, P., Eskandari, M., and Ahmadi, S.F. "Displacement potentials for functionally graded piezoelectric solids", 52, pp. 458-469 (2017).

42. Hou, P.F. and Leung, A.Y.T. "The transient responses of magneto-electro-elastic hollow cylinders", Smart Materials and Structures, 13(4), pp. 762-776 (2004).

\section{Appendix}

$$
\begin{aligned}
& \bar{X}_{1}=C_{11}^{0}\left(m_{n 1}+\beta_{1}+\beta_{2}+1\right)\left(m_{n 1}+\beta_{2}+1\right) \\
& +\left(\beta_{1} C_{12}^{0}-n^{2} C_{31}^{0}-C_{22}^{0}\right), \\
& \bar{X}_{2}=\left(\beta_{1} C_{12}^{0}-C_{31}^{0}-C_{22}^{0}\right)(i n) \\
& +\left(C_{12}^{0}+C_{31}^{0}\right)(i n)\left(m_{n 1}+\beta_{2}+1\right), \\
& \bar{X}_{3}=d_{11}^{0}\left(m_{n 1}+\beta_{1}+\beta_{2}+1\right)\left(m_{n 1}+\beta_{2}+1\right) \\
& -d_{21}^{0}\left(m_{n 1}+\beta_{2}+1\right)-n^{2} d_{31}^{0}, \\
& \bar{X}_{4}=\left(C_{31}^{0}+C_{12}^{0}\right)(i n)\left(m_{n 1}+\beta_{2}+1\right) \\
& +\left(C_{31}^{0}\left(\beta_{1}+1\right)+C_{22}^{0}\right)(i n) \\
& \bar{X}_{5}=C_{31}^{0}\left(m_{n 1}+\beta_{1}+\beta_{2}+1\right)\left(m_{n 1}+\beta_{2}+1\right) \\
& -\left(n^{2} C_{22}^{0}+\left(\beta_{1}+1\right) C_{31}^{0}\right), \\
& \bar{X}_{6}=\left(d_{31}^{0}+d_{21}^{0}\right)(i n)\left(m_{n 1}+\beta_{2}+1\right) \\
& +\left(\beta_{1}+1\right) d_{31}^{0}(i n), \\
& \bar{X}_{7}=d_{11}^{0}\left(m_{n 1}+\beta_{2}+1\right)\left(m_{n 1}+\beta_{1}+\beta_{2}+1\right) \\
& +d_{21}^{0}\left(m_{n 1}+\beta_{1}+\beta_{2}+1\right)-n^{2} d_{31}^{0}, \\
& \bar{X}_{8}=\left(d_{21}^{0}+d_{31}^{0}\right)(i n)\left(m_{n 1}+\beta_{2}+1\right) \\
& +\left(\beta_{1} d_{21}^{0}-d_{31}^{0}\right)(i n), \\
& \bar{X}_{9}=-g_{11}^{0}\left(m_{n 1}+\beta_{2}+1\right)\left(m_{n 1}+\beta_{1}+\beta_{2}+1\right)+g_{22}^{0} n^{2} \text {, } \\
& \bar{X}_{10}=C_{11}^{0}\left(m_{n 2}+\beta_{1}+\beta_{2}+1\right)\left(m_{n 2}+\beta_{2}+1\right) \\
& +\left(\beta_{1} C_{12}^{0}-C_{22}^{0}-n^{2} C_{31}^{0}\right), \\
& \bar{X}_{11}=\left(\beta_{1} C_{12}^{0}-C_{31}^{0}-C_{22}^{0}\right)(i n) \\
& +\left(C_{12}^{0}+C_{31}^{0}\right)(i n)\left(m_{n 2}+\beta_{2}+1\right),
\end{aligned}
$$




$$
\begin{aligned}
& \bar{X}_{12}=d_{11}^{0}\left(m_{n 2}+\beta_{2}+1\right)\left(m_{n 2}+\beta_{1}+\beta_{2}+1\right) \\
& +\left(\beta_{1} d_{21}^{0}-d_{31}^{0}\right)(i n) \\
& -d_{21}^{0}\left(m_{n 2}+\beta_{2}+1\right)-n^{2} d_{31}^{0}, \\
& \bar{X}_{13}=\left(C_{31}^{0}+C_{12}^{0}\right)(i n)\left(m_{n 2}+\beta_{2}+1\right) \\
& +\left(C_{31}^{0}\left(\beta_{1}+1\right)+C_{22}^{0}\right)(i n), \\
& \bar{X}_{14}=C_{31}^{0}\left(m_{n 2}+\beta_{1}+\beta_{2}+1\right)\left(m_{n 2}+\beta_{2}+1\right) \\
& -n^{2} C_{22}^{0}-\left(\beta_{1}+1\right) C_{31}^{0}, \\
& \bar{X}_{15}=\left(d_{31}^{0}+d_{21}^{0}\right)(i n)\left(m_{n 2}+\beta_{2}+1\right) \\
& +\left(\beta_{1}+1\right) d_{31}^{0}(i n) \\
& \bar{X}_{16}=d_{11}^{0}\left(m_{n 2}+\beta_{2}+1\right)\left(m_{n 2}+\beta_{1}+\beta_{2}+1\right) \\
& +d_{21}^{0}\left(m_{n 2}+\beta_{1}+\beta_{2}+1\right)-n^{2} d_{31}^{0}, \\
& \bar{X}_{17}=\left(d_{21}^{0}+e_{31}^{0}\right)(i n)\left(m_{n 2}+\beta_{2}+1\right) \\
& +\left(\beta_{1} d_{21}^{0}-d_{31}^{0}\right)(i n), \\
& \bar{X}_{18}=-g_{11}^{0}\left(m_{n 1}+\beta_{2}+1\right)\left(m_{n 2}+\beta_{1}+\beta_{2}+1\right) \\
& +n^{2} g_{22}^{0} \\
& \bar{X}_{19}=C_{11}^{0}\left(S_{n 1}+\beta_{1}+\beta_{2}+1\right)\left(S_{n 1}+\beta_{2}+1\right) \\
& +\left(\beta_{1} C_{12}^{0}-n^{2} C_{31}^{0}-C_{22}^{0}\right), \\
& \bar{X}_{20}=\left(\beta_{1} C_{12}^{0}-C_{31}^{0}-C_{22}^{0}\right)(i n) \\
& +\left(C_{12}^{0}+C_{31}^{0}\right)(i n)\left(S_{n 1}+\beta_{2}+1\right), \\
& \bar{X}_{21}=d_{11}^{0}\left(S_{n 1}+\beta_{1}+\beta_{2}+1\right)\left(S_{n 1}+\beta_{2}+1\right) \\
& -d_{21}^{0}\left(S_{n 1}+\beta_{2}+1\right)-n^{2} d_{31}^{0}, \\
& \bar{X}_{22}=\left(C_{31}^{0}+C_{12}^{0}\right)(i n)\left(S_{n 1}+\beta_{2}+1\right) \\
& +\left(C_{31}^{0}\left(\beta_{1}+1\right)+C_{22}^{0}\right)(i n), \\
& \bar{X}_{23}=C_{31}^{0}\left(S_{n 1}+\beta_{1}+\beta_{2}+1\right)\left(S_{n 1}+\beta_{2}+1\right) \\
& -n^{2} C_{22}^{0}-\left(\beta_{1}+1\right) C_{31}^{0}, \\
& \bar{X}_{24}=\left(d_{31}^{0}+d_{21}^{0}\right)(i n)\left(S_{n 1}+\beta_{2}+1\right) \\
& +\left(\beta_{1}+1\right) d_{31}^{0}(i n) \\
& \bar{X}_{25}=d_{11}^{0}\left(S_{n 1}+\beta_{2}+1\right)\left(S_{n 1}+\beta_{1}+\beta_{2}+1\right) \\
& +d_{21}^{0}\left(S_{n 1}+\beta_{1}+\beta_{2}+1\right)-n^{2} d_{31}^{0}, \\
& \bar{X}_{26}=\left(d_{21}^{0}+d_{31}^{0}\right)(\text { in })\left(S_{n 1}+\beta_{2}+1\right) \\
& \bar{X}_{27}=-g_{11}^{0}\left(S_{n 1}+\beta_{2}+1\right)\left(S_{n 1}+\beta_{1}+\beta_{2}+1\right)+n^{2} g_{22}^{0} \text {, } \\
& \bar{X}_{28}=C_{11}^{0}\left(S_{n 2}+\beta_{1}+\beta_{2}+1\right)\left(S_{n 2}+\beta_{2}+1\right) \\
& +\left(\beta_{1} C_{12}^{0}-n^{2} C_{31}^{0}-C_{22}^{0}\right), \\
& \bar{X}_{29}=\left(\beta_{1} C_{12}^{0}-C_{31}^{0}-C_{22}^{0}\right)(i n) \\
& +\left(C_{12}^{0}+C_{31}^{0}\right)(i n)\left(S_{n 2}+\beta_{2}+1\right), \\
& \bar{X}_{30}=d_{11}^{0}\left(S_{n 2}+\beta_{1}+\beta_{2}+1\right)\left(S_{n 2}+\beta_{2}+1\right) \\
& -d_{22}^{0}\left(S_{n 2}+\beta_{2}+1\right)-n^{2} d_{31}^{0}, \\
& \bar{X}_{31}=\left(C_{31}^{0}+C_{12}^{0}\right)(i n)\left(S_{n 2}+\beta_{2}+1\right) \\
& +\left(C_{31}^{0}\left(\beta_{1}+1\right)+C_{22}^{0}\right)(i n), \\
& \bar{X}_{32}=C_{31}^{0}\left(S_{n 2}+\beta_{1}+\beta_{2}+1\right)\left(S_{n 2}+\beta_{2}+1\right) \\
& -n^{2} C_{22}^{0}-\left(\beta_{1}+1\right) C_{31}^{0}, \\
& \bar{X}_{33}=\left(d_{31}^{0}+d_{21}^{0}\right)(i n)\left(S_{n 2}+\beta_{2}+1\right) \\
& +\left(\beta_{1}+1\right) d_{31}^{0}(i n) \\
& \bar{X}_{34}=d_{11}^{0}\left(S_{n 2}+\beta_{2}+1\right)\left(S_{n 2}+\beta_{1}+\beta_{2}+1\right) \\
& +d_{21}^{0}\left(S_{n 2}+\beta_{1}+\beta_{2}+1\right)-n^{2} d_{31}^{0}, \\
& \bar{X}_{35}=\left(d_{21}^{0}+d_{31}^{0}\right)(i n)\left(S_{n 2}+\beta_{2}+1\right) \\
& +\left(\beta_{1} d_{21}^{0}-d_{31}^{0}\right)(i n) \\
& \bar{X}_{36}=-g_{11}^{0}\left(S_{n 2}+\beta_{2}+1\right)\left(S_{n 2}+\beta_{1}+\beta_{2}+1\right)+n^{2} g_{22}^{0}, \\
& \bar{Y}_{1}=\left[\vartheta_{1}^{0}\left(\beta_{1}+\beta_{2}+m_{n 1}+1\right)-\vartheta_{2}^{0}\right] A_{n 1}, \\
& \bar{Y}_{2}=\vartheta_{2}^{0}(i n) A_{n 1} \text {, } \\
& \bar{Y}_{3}=-\left[q_{1}^{0}\left(\beta_{1}+\beta_{2}+m_{n 1}+1\right)+i n q_{2}^{0}\right] A_{n 1}, \\
& \bar{Y}_{4}=\left[\vartheta_{1}^{0}\left(\beta_{1}+\beta_{2}+m_{n 2}+1\right)-\vartheta_{2}^{0}\right] A_{n 2}, \\
& \bar{Y}_{5}=\vartheta_{2}^{0}(i n) A_{n 2} \text {, } \\
& \bar{Y}_{6}=-\left[q_{1}^{0}\left(\beta_{1}+\beta_{2}+m_{n 2}+1\right)+(i n) q_{2}^{0}\right] A_{n 2}, \\
& \bar{Y}_{7}=\left[\varrho_{1}^{0}\left(\beta_{1}+\beta_{2}+S_{n 1}+1\right)-\varrho_{2}^{0}\right] G_{n 1}, \\
& \bar{Y}_{8}=\varrho_{2}^{0}(i n) G_{n 1}, \\
& \bar{Y}_{9}=-\left[\gamma_{1}^{0}\left(\beta_{1}+\beta_{2}+S_{n 1}+1\right)+i n \gamma_{2}^{0}\right] G_{n 1}, \\
& \bar{Y}_{10}=\left[\varrho_{1}^{0}\left(\beta_{1}+\beta_{2}+S_{n 2}+1\right)-\varrho_{2}^{0}\right] G_{n 2}, \\
& \bar{Y}_{11}=\varrho_{2}^{0}(i n) G_{n 2},
\end{aligned}
$$




$$
\begin{aligned}
& \bar{Y}_{12}=-\left[\gamma_{1}^{0}\left(\beta_{1}+\beta_{2}+S_{n 1}+1\right)+i n \gamma_{2}^{0}\right] G_{n 2}, \\
& \bar{Y}_{1}^{0}=\left[\vartheta_{1}^{0}\left(\beta_{1}+\beta_{2}+m_{01}+1\right)-\mu_{2}^{0}\right] A_{01}, \\
& \bar{X}_{1}^{0}=C_{11}^{0}\left(m_{01}+\beta_{1}+\beta_{2}+1\right)\left(m_{01}+\beta_{2}+1\right) \\
& +\left(\beta_{1} C_{12}^{0}-C_{22}^{0}\right) \\
& \bar{X}_{2}^{0}=d_{11}^{0}\left(m_{01}+\beta_{1}+\beta_{2}+1\right)\left(m_{01}+\beta_{2}+1\right) \\
& -d_{21}^{0}\left(m_{01}+\beta_{2}+1\right), \\
& \bar{X}_{3}^{0}=d_{11}^{0}\left(m_{01}+\beta_{2}+1\right)\left(m_{01}+\beta_{1}+\beta_{2}+1\right) \\
& +d_{21}^{0}\left(m_{01}+\beta_{1}+\beta_{2}+1\right), \\
& \bar{X}_{4}^{0}=-g_{11}^{0}\left(m_{01}+\beta_{2}+1\right)\left(\beta_{1}+\beta_{2}+m_{01}+1\right) \text {, } \\
& \bar{X}_{5}^{0}=C_{11}^{0}\left(m_{02}+\beta_{1}+\beta_{2}+1\right)\left(m_{02}+\beta_{2}+1\right) \\
& +\left(\beta_{1} C_{12}^{0}-C_{22}^{0}\right) \\
& \bar{X}_{6}^{0}=d_{11}^{0}\left(m_{02}+\beta_{2}+1\right)\left(m_{02}+\beta_{1}+\beta_{2}+1\right) \\
& -d_{21}^{0}\left(m_{02}+\beta_{2}+1\right), \\
& \bar{X}_{7}^{0}=d_{11}^{0}\left(m_{02}+\beta_{2}+1\right)\left(m_{02}+\beta_{1}+\beta_{2}+1\right) \\
& +d_{21}^{0}\left(m_{02}+\beta_{1}+\beta_{2}+1\right), \\
& \bar{X}_{8}^{0}=-g_{11}^{0}\left(m_{01}+\beta_{2}+1\right)\left(m_{02}+\beta_{1}+\beta_{2}+1\right), \\
& \bar{X}_{9}^{0}=C_{11}^{0}\left(S_{01}+\beta_{1}+\beta_{2}+1\right)\left(S_{01}+\beta_{2}+1\right) \\
& +\left(\beta_{1} C_{12}^{0}-C_{22}^{0}\right) \\
& \bar{X}_{10}^{0}=d_{11}^{0}\left(S_{01}+\beta_{1}+\beta_{2}+1\right)\left(S_{01}+\beta_{2}+1\right) \\
& -d_{21}^{0}\left(S_{01}+\beta_{2}+1\right), \\
& \bar{X}_{11}^{0}=d_{11}^{0}\left(S_{01}+\beta_{2}+1\right)\left(S_{01}+\beta_{1}+\beta_{2}+1\right) \\
& +d_{21}^{0}\left(S_{01}+\beta_{1}+\beta_{2}+1\right), \\
& \bar{X}_{12}^{0}=-g_{11}^{0}\left(S_{01}+\beta_{2}+1\right)\left(S_{01}+\beta_{1}+\beta_{2}+1\right), \\
& \bar{X}_{13}^{0}=C_{11}^{0}\left(S_{02}+\beta_{1}+\beta_{2}+1\right)\left(S_{02}+\beta_{2}+1\right) \\
& +\left(\beta_{1} C_{12}^{0}-C_{22}^{0}\right), \\
& \bar{X}_{14}^{0}=d_{11}^{0}\left(S_{02}+\beta_{1}+\beta_{2}+1\right)\left(S_{02}+\beta_{2}+1\right) \\
& -d_{22}^{0}\left(S_{02}+\beta_{2}+1\right), \\
& \bar{X}_{15}^{0}=d_{11}^{0}\left(S_{02}+\beta_{2}+1\right)\left(S_{02}+\beta_{1}+\beta_{2}+1\right) \\
& +d_{21}^{0}\left(S_{02}+\beta_{1}+\beta_{2}+1\right) \\
& \bar{X}_{16}^{0}=-g_{11}^{0}\left(S_{02}+\beta_{2}+1\right)\left(S_{02}+\beta_{1}+\beta_{2}+1\right), \\
& \bar{Y}_{2}^{0}=-\left[q_{1}^{0}\left(\beta_{1}+\beta_{2}+m_{01}+1\right)\right] A_{01}, \\
& \bar{Y}_{3}^{0}=\left[\vartheta_{1}^{0}\left(\beta_{1}+\beta_{2}+m_{02}+1\right)-\vartheta_{2}^{0}\right] A_{02}, \\
& \bar{Y}_{4}^{0}=-\left[q_{1}^{0}\left(\beta_{1}+\beta_{2}+m_{02}+1\right)\right] A_{02}, \\
& \bar{Y}_{5}^{0}=\left[\varrho_{1}^{0}\left(\beta_{1}+\beta_{2}+S_{01}+1\right)-\varrho_{2}^{0}\right] G_{01}, \\
& \bar{Y}_{6}^{0}=-\left[\gamma_{1}^{0}\left(\beta_{1}+\beta_{2}+S_{01}+1\right)\right] G_{01}, \\
& \bar{Y}_{7}^{0}=\left[\varrho_{1}^{0}\left(\beta_{1}+\beta_{2}+S_{02}+1\right)-\varrho_{2}^{0}\right] G_{02}, \\
& \bar{Y}_{8}^{0}=-\left[\gamma_{1}^{0}\left(\beta_{1}+\beta_{2}+S_{01}+1\right)\right] G_{02}, \\
& Z_{n j}^{1}=C_{11}^{0} \zeta_{n j}+C_{12}^{0}\left(i n L_{n j}+1\right)+d_{11}^{0} P_{n j} \zeta_{n j} \text {, } \\
& Z_{n j}^{2}=C_{12}^{0} \zeta_{n j}+C_{22}^{0}\left(i n L_{n j}+1\right)+d_{21}^{0} P_{n j} \zeta_{n j}, \\
& Z_{n j}^{3}=i n C_{31}^{0}+C_{31}^{0} L_{n j}\left(\zeta_{n j}-1\right)+i n d_{31}^{0} P_{n j}, \\
& Z_{n j}^{4}=d_{11}^{0} \zeta_{n j}+d_{21}^{0}\left(i n L_{n j}+1\right)-g_{11}^{0} P_{n j} \zeta_{n j}, \\
& Z_{n j}^{5}=i n d_{31}^{0}+d_{31}^{0} L_{n j}\left(\zeta_{n j}-1\right)-i n g_{22}^{0} P_{n j}, \\
& Z_{0 j}^{1}=C_{11}^{0} \zeta_{0 j}+C_{12}^{0}+d_{11}^{0} P_{0 j} \zeta_{0 j}, \\
& Z_{0 j}^{2}=C_{12}^{0} \zeta_{0 j}+C_{22}^{0}+d_{21}^{0} P_{0 j} \zeta_{0 j}, \\
& Z_{n j}^{3}=C_{31}^{0}\left(\zeta_{0 j}-1\right), \\
& Z_{0 j}^{4}=d_{11}^{0} \zeta_{0 j}+d_{21}^{0}-g_{11}^{0} P_{0 j} \zeta_{0 j}, \\
& Z_{0 j}^{5}=d_{31}^{0}\left(\zeta_{0 j}-1\right), \\
& \bar{W}_{n}^{1}=C_{11}^{0}\left(m_{n 1}+\beta_{2}+1\right) Q_{n}^{1}+C_{12}^{0}\left(Q_{n}^{1}+i n Q_{n}^{5}\right) \\
& +d_{11}^{0}\left(m_{n 1}+\beta_{2}+1\right) Q_{n}^{9}-\vartheta_{1}^{0} A_{n 1}, \\
& \bar{W}_{n}^{2}=C_{11}^{0}\left(m_{n 2}+\beta_{2}+1\right) Q_{n}^{2}+C_{12}^{0}\left(Q_{n}^{2}+i n Q_{n}^{6}\right) \\
& +d_{11}^{0}\left(m_{n 2}+\beta_{2}+1\right) Q_{n}^{10}-\vartheta_{1}^{0} A_{n 2}, \\
& \bar{W}_{n}^{3}=C_{11}^{0}\left(S_{n 1}+\beta_{2}+1\right) Q_{n}^{3}+C_{12}^{0}\left(Q_{n}^{3}+i n Q_{n}^{7}\right) \\
& +d_{11}^{0}\left(S_{n 1}+\beta_{2}+1\right) Q_{n}^{11}-\varrho_{1}^{0} G_{n 1}, \\
& \bar{W}_{n}^{4}=C_{11}^{0}\left(S_{n 2}+\beta_{2}+1\right) Q_{n}^{4}+C_{12}^{0}\left(Q_{n}^{4}+i n Q_{n}^{8}\right) \\
& +d_{11}^{0}\left(S_{n 2}+\beta_{2}+1\right) Q_{n}^{12}-\varrho_{1}^{0} G_{n 2}, \\
& \bar{W}_{n}^{5}=C_{12}^{0}\left(m_{n 1}+\beta_{2}+1\right) Q_{n}^{1}+C_{22}^{0}\left(Q_{n}^{1}+i n Q_{n}^{5}\right) \\
& +d_{21}^{0}\left(m_{n 1}+\beta_{2}+1\right) Q_{n}^{9}-\vartheta_{2}^{0} A_{n 1}, \\
& \bar{W}_{n}^{6}=C_{12}^{0}\left(m_{n 2}+\beta_{2}+1\right) Q_{n}^{2}+C_{22}^{0}\left(Q_{n}^{2}+i n Q_{n}^{6}\right) \\
& +d_{21}^{0}\left(m_{n 2}+\beta_{2}+1\right) Q_{n}^{10}-\vartheta_{2}^{0} A_{n 2},
\end{aligned}
$$




$$
\begin{aligned}
& \bar{W}_{n}^{7}=C_{12}^{0}\left(S_{n 1}+\beta_{2}+1\right) Q_{n}^{3}+C_{22}^{0}\left(Q_{n}^{3}+i n Q_{n}^{7}\right) \\
& +d_{21}^{0}\left(S_{n 1}+\beta_{2}+1\right) Q_{n}^{11}-\varrho_{2}^{0} G_{n 1}, \\
& \bar{W}_{n}^{8}=C_{12}^{0}\left(S_{n 2}+\beta_{2}+1\right) Q_{n}^{4}+C_{22}^{0}\left(Q_{n}^{4}+i n Q_{n}^{8}\right) \\
& +d_{21}^{0}\left(S_{n 2}+\beta_{2}+1\right) Q_{n}^{12}-\varrho_{2}^{0} G_{n 2}, \\
& \bar{W}_{n}^{9}=i n C_{31}^{0} Q_{n}^{1}+C_{31}^{0}\left(m_{n 1}+\beta_{2}\right) Q_{n}^{5}+i n d_{31}^{0} Q_{n}^{9}, \\
& \bar{W}_{n}^{10}=i n C_{31}^{0} Q_{n}^{2}+C_{31}^{0}\left(m_{n 2}+\beta_{2}\right) Q_{n}^{6}+i n d_{31}^{0} Q_{n}^{10}, \\
& \bar{W}_{n}^{11}=i n C_{31}^{0} Q_{n}^{3}+C_{31}^{0}\left(S_{n 1}+\beta_{2}\right) Q_{n}^{7}+i n d_{31}^{0} Q_{n}^{11}, \\
& \bar{W}_{n}^{12}=i n C_{31}^{0} Q_{n}^{4}+C_{31}^{0}\left(S_{n 2}+\beta_{2}\right) Q_{n}^{8}+i n d_{31}^{0} Q_{n}^{12}, \\
& \bar{W}_{n}^{13}=d_{11}^{0}\left(m_{n 1}+\beta_{2}+1\right) Q_{n}^{1}+d_{21}^{0}\left(Q_{n}^{1}+i n Q_{n}^{5}\right) \\
& -g_{11}^{0}\left(m_{n 1}+\beta_{2}+1\right) Q_{n}^{9}+q_{1}^{0} A_{n 1}, \\
& \bar{W}_{n}^{14}=d_{11}^{0}\left(m_{n 2}+\beta_{2}+1\right) Q_{n}^{2}+d_{21}^{0}\left(Q_{n}^{2}+i n Q_{n}^{6}\right) \\
& -g_{11}^{0}\left(m_{n 2}+\beta_{2}+1\right) Q_{n}^{10}+q_{1}^{0} A_{n 2}, \\
& \bar{W}_{n}^{15}=d_{11}^{0}\left(S_{n 1}+\beta_{2}+1\right) Q_{n}^{3}+d_{21}^{0}\left(Q_{n}^{3}+i n Q_{n}^{7}\right) \\
& -g_{11}^{0}\left(S_{n 1}+\beta_{2}+1\right) Q_{n}^{11}+\gamma_{1}^{0} G_{n 1}, \\
& \bar{W}_{n}^{16}=d_{11}^{0}\left(S_{n 2}+\beta_{2}+1\right) Q_{n}^{4}+d_{21}^{0}\left(Q_{n}^{4}+i n Q_{n}^{8}\right) \\
& -g_{11}^{0}\left(S_{n 2}+\beta_{2}+1\right) Q_{n}^{12}+\gamma_{1}^{0} G_{n 2}, \\
& \bar{W}_{n}^{17}=i n d_{31}^{0} Q_{n}^{1}+d_{31}^{0}\left(m_{n 1}+\beta_{2}\right) Q_{n}^{5}-i n g_{22}^{0} Q_{n}^{9} \\
& +q_{2}^{0} A_{n 1}, \\
& \bar{W}_{n}^{18}=i n d_{31}^{0} Q_{n}^{2}+d_{31}^{0}\left(m_{n 2}+\beta_{2}\right) Q_{n}^{6}-i n g_{22}^{0} Q_{n}^{10} \\
& +q_{2}^{0} A_{n 2}, \\
& \bar{W}_{n}^{19}=i n d_{31}^{0} Q_{n}^{3}+d_{31}^{0}\left(S_{n 1}+\beta_{2}\right) Q_{n}^{7}-i n g_{22}^{0} F_{n}^{11} \\
& +\gamma_{2}^{0} G_{n 1}, \\
& \bar{W}_{n}^{20}=i n d_{31}^{0} Q_{n}^{4}+d_{31}^{0}\left(S_{n 2}+\beta_{2}\right) Q_{n}^{8}-i n g_{22}^{0} Q_{n}^{12} \\
& +\gamma_{2}^{0} G_{n 2}, \\
& \bar{W}_{0}^{1}=C_{11}^{0}\left(m_{01}+\beta_{2}+1\right) Q_{0}^{1}+C_{12}^{0} Q_{0}^{1} \\
& +d_{11}^{0}\left(m_{01}+\beta_{2}+1\right) Q_{0}^{9}-\vartheta_{1}^{0} A_{01}, \\
& \bar{W}_{0}^{2}=C_{11}^{0}\left(m_{02}+\beta_{2}+1\right) Q_{0}^{2}+C_{12}^{0} Q_{0}^{2} \\
& +d_{11}^{0}\left(m_{01}+\beta_{2}+1\right) Q_{0}^{10}-\vartheta_{1}^{0} A_{02},
\end{aligned}
$$

$$
\begin{aligned}
& \bar{W}_{0}^{3}= C_{11}^{0}\left(S_{01}+\beta_{2}+1\right) Q_{0}^{3}+C_{12}^{0} Q_{0}^{3} \\
&+d_{11}^{0}\left(S_{01}+\beta_{2}+1\right) Q_{0}^{11}-\varrho_{1}^{0} G_{01}, \\
& \bar{W}_{0}^{4}= C_{11}^{0}\left(S_{02}+\beta_{2}+1\right) Q_{0}^{4}+C_{12}^{0} Q_{0}^{4} \\
&+d_{11}^{0}\left(S_{02}+\beta_{2}+1\right) Q_{0}^{12}-\varrho_{1}^{0} G_{02}, \\
& \bar{W}_{0}^{5}= C_{12}^{0}\left(m_{01}+\beta_{2}+1\right) Q_{0}^{1}+C_{22}^{0} Q_{0}^{1} \\
&+d_{21}^{0}\left(m_{01}+\beta_{2}+1\right) Q_{0}^{9}-\vartheta_{2}^{0} A_{01}, \\
& \bar{W}_{0}^{6}= C_{12}^{0}\left(m_{02}+\beta_{2}+1\right) Q_{0}^{2}+C_{22}^{0} Q_{0}^{2} \\
&+d_{21}^{0}\left(m_{02}+\beta_{2}+1\right) Q_{0}^{10}-\vartheta_{2}^{0} A_{02}, \\
& \bar{W}_{0}^{7}= C_{12}^{0}\left(S_{01}+\beta_{2}+1\right) Q_{0}^{3}+C_{22}^{0} Q_{0}^{3} \\
&+d_{21}^{0}\left(S_{01}+\beta_{2}+1\right) Q_{0}^{11}-\varrho_{2}^{0} G_{01}, \\
& \bar{W}_{0}^{8}= C_{12}^{0}\left(S_{02}+\beta_{2}+1\right) Q_{0}^{4}+C_{22}^{0} Q_{0}^{4} \\
&+d_{21}^{0}\left(S_{02}+\beta_{2}+1\right) Q_{0}^{12}-\varrho_{2}^{0} G_{02}, \\
& \bar{W}_{0}^{9}= d_{11}^{0}\left(m_{01}+\beta_{2}+1\right) Q_{0}^{1}+d_{21}^{0} F_{0}^{1} \\
&-g_{11}^{0}\left(m_{01}+\beta_{2}+1\right) Q_{0}^{9}+q_{1}^{0} A_{01}, \\
& \bar{W}_{0}^{10}= d_{11}^{0}\left(m_{02}+\beta_{2}+1\right) Q_{0}^{2}+d_{21}^{0} Q_{0}^{2} \\
&-g_{11}^{0}\left(m_{02}+\beta_{2}+1\right) Q_{0}^{10}+q_{1}^{0} A_{02}, \\
& \bar{W}_{0}^{11}= d_{11}^{0}\left(S_{01}+\beta_{2}+1\right) Q_{0}^{3}+d_{21}^{0} Q_{0}^{3} \\
&-g_{11}^{0}\left(S_{01}+\beta_{2}+1\right) Q_{0}^{11}+\gamma_{1}^{0} G_{01}, \\
& \bar{W}_{11}^{0}\left(S_{02}+\beta_{2}+1\right) Q_{0}^{4}+d_{21}^{0} Q_{0}^{4} \\
&\left.-\beta_{2}+1\right) Q_{0}^{12}+\gamma_{1}^{0} G_{02}, \\
&
\end{aligned}
$$

\section{Biographies}

Mehdi Gharibavi is a $\mathrm{PhD}$ candidate at East China University of Science and Technology, Shanghai, P.R. China. He received his MSc degree in Mechanical Engineering from Payame Noor University (PNU) in Tehran, Iran, in 2015 and BSc degree from Islamic Azad University, Qazvin, Iran, in 2011. His current research interests include control and stability of mechanical systems, intelligent control and its industrial applications, and medical device.

Jianjun Yi is a Professor at East China University of Science and Technology, Shanghai, P.R. China. Professor YI received his PhD in 1999, MSc in 1996, and BSc in 1991 in Mechanical Manufacturing and 
Automation from Dalian University of Technology (DLUT), P.R. China. He worked as a post-doctoral fellow in the School of Mechanical Science and Engineering, Huazhong University of Science and Technology (HUST), from March 1999 to March 2001 and in the Institute of Artificial Intelligence (AI), Zhejiang University (ZJU) from January 2005 to March 2007. Also, he worked as a guest Professor at the Technical University of Munich (TUM), Germany, from September 2005 to October 2007. Professor $\mathrm{Yi}$ is a Member of the IEEE and a senior member of the China Mechanical Engineering Society. His current research interests include digital control of mechatronic systems, intelligent control and its industrial applications, distributed real-time control systems and embedded systems, RFID and internet of things, automotive electronics, and ontology for knowledge management. 